\title{
REGULARITY OF DISPLACEMENT SOLUTIONS IN HENCKY PLASTICITY. I: THE EXTREMAL RELATION
}

Abstract. The aim of this paper is to study the problem of regularity of displacement solutions in Hencky plasticity. A non-homogeneous material whose elastic-plastic properties change discontinuously is considered. We find (in an explicit form) the extremal relation between the displacement formulation (defined on the space of bounded deformation) and the stress formulation of the variational problem in Hencky plasticity. This extremal relation is used in the proof of the regularity of displacements.

In part II of the paper, we will prove that the displacement solution belongs to the classical Sobolev space (if the stress solution belongs to the interior of a set of admissible stresses, at each point). We will find the regularity theorem for displacement solutions in composite materials whose elastic-plastic properties may change discontinuously.

1. Introduction. The principal aim of this paper is to prove regularity of displacement solutions in Hencky plasticity. Here, a non-homogeneous (composite) material is considered, whose elastic-plastic properties change discontinuously. The extremal relation between the displacement formulation defined on $B D(\Omega)$ and the stress formulation of the relevant variational problem is found (see (3.1), (3.2) and Section 2). This relation is used in the proof of the regularity of solutions. The basic results of the first part of this paper are presented at the end of the fourth section (Theorems 8 and 9).

In the second part of the paper, we prove that the displacement solution belongs to the space $L D(\Omega)$ (if the stress solution belongs to the interior of a set of admissible stresses, at each point). Moreover, under the aforemen-

2010 Mathematics Subject Classification: Primary 49N60; Secondary 49J45, 49K30, 74C05. Key words and phrases: duality in convex optimization for Hencky plasticity, bounded deformation, boundary transmission problems in plasticity, composite materials. 
tioned assumption, the relaxed Dirichlet condition is satisfied exactly by the displacement solution for the non-homogeneous material.

The study of the regularity of displacement solutions is significant for understanding the appearance of cracks. If the set of admissible hydrostatic stresses $\boldsymbol{\sigma}$ is bounded from above (i.e., for some $\delta>0, \sum_{i=1}^{3} \sigma_{i i}=\operatorname{tr} \boldsymbol{\sigma} \leq$ $\delta<\infty$ for every admissible $\boldsymbol{\sigma})$ the arising and increase of cracks is possible in an elastic-plastic material. Indeed, it is sufficient to analyze the relaxed Dirichlet condition (see [8, 9] and also [10]). Let us recall that in a model of soil materials the set of admissible hydrostatic stresses is bounded from above. Note that when locating cracks, the non-homogeneity of the elasticplastic potential is especially important (see [3]). Gold, a homogeneous metal (which lacks micro-defects) is at the same time perfectly plastic.

In [1] (see also 4]) the existence of solutions for the nonlinear elastic potential is proved in the space $S B V(\Omega)$ of special vector fields with bounded variation $(S B D(\Omega)$ of special vector fields with bounded deformation, respectively). The authors assume that the potentials considered have nonlinear behavior at infinity.

In [21] the problem of regularity of displacement solutions is investigated in the case of an isotropic Hencky material using the von Mises yield criterion (cf. [21, Lemma 2.2]). The proof of the main theorem of [21] (Theorem 5.1) is based on the relation between the displacement field and the associated stress tensor (cf. formula (1.8) of [21]). However, the formula (1.8) describes the relation between the displacement solution and the stress solution only in the case when the space of admissible stress fields is given by the inequality $\left(\sum_{i, j=1}^{n}\left|\sigma_{i j}^{D}\right|^{2}\right)^{1 / 2} \leq k$ (see the Prandtl-Reuss law of plasticity [25, formula (2.10b)]). Therefore, these authors do not consider the Tresca yield criterion or the yield criterion of soil material (where the set of admissible stresses is a convex cone). Moreover, they do not consider bodies clamped at the boundary.

Anzellotti and Giaquinta [3] study the local regularity of minimizers of functionals defined on the space $B V(\Omega)$. They obtain the regularity of minimizers under the assumption that the normal integrand

$$
\Omega \times \mathbb{R}^{n \times n} \ni(x, \mathbf{p}) \mapsto j(x, \mathbf{p}) \in \mathbb{R} \cup\{+\infty\}
$$

is of class $C^{2}$ with respect to $\mathbf{p}$, and is continuous with respect to the first variable. These authors do not consider boundary conditions.

Seregin [35] investigates the local continuity of stress and displacement solution in a homogeneous Hencky material under the assumption of regularity of the volume forces. He considers the original problem only for displacements which exactly satisfy the boundary condition. Therefore, the relaxation of the displacement boundary condition is not studied. 
In [7] the problem of regularity of displacement solutions is considered for the case of a non-homogeneous Hencky material whose elastic-plastic properties may change discontinuously. If the stress solution is continuous and belongs to the interior of the set of admissible stresses, then the regularity of displacements solutions is obtained. However, the stress solution is discontinuous (see [38]).

Liu [26] investigates the regularity of a weak solution of a quasi-linear elliptic transmission problem with two subdomains. He assumes that the interface is smooth and does not intersect the exterior boundary of $\Omega$. Only elastic (or non-linearly elastic) problems are considered. Indeed, the relation between stresses $\boldsymbol{\sigma}$ and displacements $\mathbf{u}$ is given by

$$
\boldsymbol{\sigma}=k(z,|\nabla \mathbf{u}|) \nabla \mathbf{u} .
$$

Moreover

$$
k(z, t) \geq M(t+1)^{p-2} \quad \text { for every } t>0,
$$

where $1<p<\infty$ and $M>0$ (see assumptions (R1) and (RR2)). Then $k(z, t) \geq M(t+1)^{-1+\delta}$ for some $\delta>0$. Therefore

$$
k(z, t) t \geq M_{1} t^{\delta} \quad \text { for every } t>1,
$$

for some $M_{1}>0$ and $\delta>0$. But this conflicts with the assumption that admissible stresses $\boldsymbol{\sigma}$ are bounded (except the hydrostatic part) in plasticity. Moreover, in [26] the author obtained the existence of solutions for an arbitrarily strong and steady force (the limit analysis is omitted).

In [14, 22, 23, 27, 30, 31, 32, 33, the regularity of solutions of quasilinear (or linear) elliptic boundary transmission problems of a domain $\Omega$ (composed of a finite family of regular subdomains $\Omega_{i}$ ) is studied. In each subdomain $\Omega_{i}$ the coefficients of the equations are either constant or smooth.

In [30, 31] the authors study transmission problems for elliptic operators of order $2 m$ with general boundary and interface conditions. They obtain regularity and asymptotics of solutions in ordinary Sobolev spaces for nonconstant coefficient operators. In the case of two-dimensional domains, they assume that the boundaries of subdomains are Lipschitz-continuous and are smooth curvilinear polygons. In the case of three-dimensional domains, the boundaries of subdomains are supposed to be smooth (except at one point).

In 33 the author investigates the regularity of solutions of interface problems for the Laplacian in two dimensions. The regularity of the results is independent of global bounds of the data. The data considered are constant on subdomains and they could be interpreted as a diffusion term.

In [27] the transmission problems for the Laplace operator in a twodimensional domain are investigated. The regularity of the solutions obtained is better than $H^{1}$. 
The transmission problems for elasticity operators are studied in [22, 23, 32. The domain considered is divided into polyhedral subdomains in [22, 23]. Moreover, the author assumes that the growth properties of the differential operator may vary from subdomain to subdomain.

In [16] the transmission problem in a domain composed of two polyhedral subdomains (with a plane interface) is investigated. It is essential for the proof that the energy densities satisfy certain growth properties.

In [28] the authors study the Sobolev regularity of the solution of the transmission problem in a polygonal domain of the plane, with unilateral boundary conditions of Signorini type on part of the boundary and Dirichlet or Neumann boundary conditions on the remainder part.

In [13 the problem of regularity of solutions for a static plate is studied.

Kohn and Temam 24 solve the existence problem for an elastic-perfectly plastic solid made of a homogeneous and isotropic Hencky material. To prove that the functional of the total potential energy is weakly* lower semicontinuous (l.s.c.) in the space $B D(\Omega)$, the method of relaxation of the kinematic boundary condition is used (see also [36]).

The existence problem for an anisotropic elastic-perfectly plastic solid made of a non-homogeneous Hencky material, with the Signorini constraints on the boundary, is solved in [5]. The Signorini problem for an isotropic and homogeneous body made of a Hencky material with the von Mises plastic yield condition is solved in [39].

We show in 8 ] that the relaxation of the problems defined in [24] and [5] is the l.s.c. regularization of the displacement energy (cf. [17]). That is, the relaxation given in [24] and [5] is the largest (l.s.c. in weak* $B D(\Omega)$ topology) minorant less than the displacement energy functional of the original problem.

In this paper we find explicitly the extremal relation between the displacement formulation and the stress formulation of the variational problem in Hencky plasticity. The dual problem is presented in [38], in the case where the original (displacement) problem is defined on the space $L D(\Omega)$. But the existence of solutions for the bidual problem is proved in the space $B D(\Omega)$ (or $U(\Omega) \equiv\left\{\mathbf{u} \in B D(\Omega) \mid \operatorname{div} \mathbf{u} \in L^{2}(\Omega)\right\}$ ) and $L D(\Omega) \subsetneq B D(\Omega)$ (see [24], [36], [5]). In [36] the extremal relation for a homogeneous body is considered; however, the definition of the scheme of duality is omitted (the spaces $Y$ and $V^{*}$ are not provided there, see Section 2). Moreover, in [36] the author studies the duality between the generalized stresses and strains but does not derive the dual or bidual problem from the original problem, when the original problem is defined on $B D(\Omega)$.

The extremal relation under consideration determines the relation between the displacement solution and the stress solution. It is a substitute 
for the elastic law in the theory of elasticity. Unfortunately, in the theory of plasticity, the displacement formulation is relaxed. Therefore, we obtain only a global relation between the displacement solution and the stress solution.

We consider any given yield criterion (von Mises, Tresca or yield criterion of soil material) in both parts of this paper. However, we have to assume in part II that the stress solution belongs to the space $W^{n}(\Omega$, div) (see (3.7)).

In part II we prove the regularity of displacement solutions. We do not assume the continuity of the displacement field on the interface between subdomains because the space $B D(\Omega)$ contains discontinuous functions. The elastic-plastic potential is a normal integrand (see [17, Chapter 8, p. 232] and Definition 1); thus, this potential is a discontinuous function with respect to the space variable in the case of a non-homogenized body composed of a few components. Moreover, the yield criterion may change in a discontinuous way, i.e., the yield criterion may jump on the interface between subdomains.

The problem of the regularity of displacement solutions has not been discussed in the papers [3, 35] in the case of discontinuous elastic-plastic properties. Moreover, the regularity of solutions in [21] was proved only for the Mises yield criterion. Therefore the set of admissible hydrostatic stresses is not bounded in [21].

2. Duality in convex optimization. In this section we introduce a family of perturbations of the original problem which will be studied later (cf. Chapter 3 of [17]).

Let $V$ and $V^{*}\left(Y\right.$ and $\left.Y^{*}\right)$ be two topological vector spaces placed in duality by the bilinear pairing $\langle\cdot, \cdot\rangle_{V}\left(\langle\cdot, \cdot\rangle_{Y}\right.$, respectively). We shall assume the existence of a continuous linear operator $\Lambda$ from $V$ into $Y$, with adjoint $\Lambda^{*}$. Considering a function $V \ni \mathbf{v} \mapsto F(\mathbf{v})+G(\Lambda \mathbf{v}) \in \mathbb{R} \cup\{+\infty\}$, we are concerned with the minimization problem

$(P) \quad \inf \{F(\mathbf{v})+G(\Lambda \mathbf{v}) \mid \mathbf{v} \in V\}$.

We shall also consider a function $\Phi: V \times Y \rightarrow \mathbb{R} \cup\{+\infty\}$ such that $\Phi(\mathbf{v}, \mathbf{0})=F(\mathbf{v})+G(\Lambda \mathbf{v})$, and for every $\mathbf{z} \in Y$ we shall consider the minimization problem

$$
\left(P_{\mathbf{z}}\right) \quad \inf \{\Phi(\mathbf{v}, \mathbf{z}) \mid \mathbf{v} \in V\},
$$

where $\Phi(\mathbf{v}, \mathbf{z}) \equiv F(\mathbf{v})+G(\Lambda(\mathbf{v})+\mathbf{z})$ for every $\mathbf{v} \in V$ and $\mathbf{z} \in Y$. The problems $\left(P_{\mathbf{z}}\right)$ are called perturbed problems of $(P)$. Let $\Phi^{*}$ be the conjugate function of $\Phi$ in the duality between $V \times Y$ and $V^{*} \times Y^{*}$, given by

$$
\begin{aligned}
\Phi^{*}\left(\mathbf{v}^{*}, \mathbf{z}^{*}\right)=\sup \left\{\left\langle\mathbf{v}^{*}, \mathbf{v}\right\rangle_{V}-F(\mathbf{v})-\left\langle\mathbf{z}^{*}, \Lambda \mathbf{v}\right\rangle_{Y}+\left\langle\mathbf{z}^{*}, \Lambda(\mathbf{v})+\mathbf{z}\right\rangle_{Y}\right. \\
-G(\Lambda(\mathbf{v})+\mathbf{z}) \mid \mathbf{v} \in V, \mathbf{z} \in Y\}=F^{*}\left(\mathbf{v}^{*}-\Lambda^{*} \mathbf{z}^{*}\right)+G^{*}\left(\mathbf{z}^{*}\right) .
\end{aligned}
$$


The problem

$$
\left(P^{*}\right) \sup \left\{-\Phi^{*}\left(\mathbf{0}, \mathbf{z}^{*}\right) \mid \mathbf{z}^{*} \in Y^{*}\right\}
$$

is called the dual problem of $(P)$ with respect to $\Phi$.

It is natural to associate the perturbed problems $\left(\mathbf{v}^{*} \in V^{*}\right)$

$$
\left(P_{\mathbf{v}^{*}}^{*}\right) \quad \sup \left\{-\Phi^{*}\left(\mathbf{v}^{*}, \mathbf{z}^{*}\right) \mid \mathbf{z}^{*} \in Y^{*}\right\}
$$

with the dual problem $\left(P^{*}\right)$, and to determine the dual problem of $\left(P^{*}\right)$ with respect to these perturbations; we easily arrive at the following problem which will be called the bidual problem of $(P)$ :

$$
\left(P^{* *}\right) \quad \inf \left\{\Phi^{* *}(\mathbf{v}, \mathbf{0}) \mid \mathbf{v} \in V\right\},
$$

where $\Phi^{* *}(\mathbf{v}, \mathbf{z})=F^{* *}(\mathbf{v})+G^{* *}(\Lambda(\mathbf{v})+\mathbf{z})$ for every $\mathbf{v} \in V, \mathbf{z} \in Y$ and

$$
\begin{aligned}
F^{* *}(\mathbf{v}) & =\sup \left\{\left\langle\mathbf{v}^{*}, \mathbf{v}\right\rangle_{V}-F^{*}\left(\mathbf{v}^{*}\right) \mid \mathbf{v}^{*} \in V^{*}\right\}, \\
G^{* *}(\Lambda(\mathbf{v})+\mathbf{z}) & =\sup \left\{\left\langle\mathbf{z}^{*}, \Lambda(\mathbf{v})+\mathbf{z}\right\rangle_{Y}-G^{*}\left(\mathbf{z}^{*}\right) \mid \mathbf{z}^{*} \in Y^{*}\right\} .
\end{aligned}
$$

3. Some basic definitions and theorems. Let $\Omega$ be a bounded open, connected set of class $C^{1}$ in $\mathbb{R}^{n}$. The space of continuous functions with compact support is denoted by $C_{c}$. Let $C^{\infty}\left(\Omega, \mathbb{R}^{m}\right)$ be the space of $\mathbb{R}^{m}$ valued, infinitely differentiable functions. Moreover, the space of infinitely differentiable functions equal to 0 at the boundary $\operatorname{Fr} \Omega$ of $\Omega$ is denoted by $C_{0}^{\infty}(\Omega)$. Finally, $\mathbb{M}_{b}\left(\Omega, \mathbb{R}^{m}\right)$ is the space of $\mathbb{R}^{m}$-valued, bounded, regular measures on $\Omega$, with the norm $\|\cdot\|_{\mathbb{M}_{b}\left(\Omega, \mathbb{R}^{m}\right)}$.

In the main part of this paper we will use one of the duality pairs $\left(\mathbb{M}_{r}, C_{c}\right)$ or $\left(\mathbb{M}_{b}, C_{0}\right)$, where $\mathbb{M}_{r}$ is the space of regular Radon measures. Bilinear forms will be denoted by brackets $\langle\cdot, \cdot\rangle$, and the scalar product of $\mathbf{z}, \mathbf{z}^{*} \in \mathbb{R}^{n}$ by $\mathbf{z} \cdot \mathbf{z}^{*}$ or $\mathbf{z z}^{*}$. The scalar product of $\mathbf{w}$ and $\mathbf{w}^{*} \in \mathbb{R}^{n \times n}$ is denoted by $\mathbf{w}: \mathbf{w}^{*}=w^{i j} w_{i j}^{*}$. Let $\mathbf{g}=\left(g_{1}, \ldots, g_{m}\right) \in C\left(\bar{\Omega}, \mathbb{R}^{m}\right)$ and $\boldsymbol{\mu}=\left(\mu_{1}, \ldots, \mu_{m}\right) \in$ $\mathbb{M}_{b}\left(\Omega, \mathbb{R}^{m}\right)$. Then $\int_{\Omega} \mathbf{g} \cdot \boldsymbol{\mu}=\int_{\Omega} \mathbf{g} \boldsymbol{\mu} \equiv \sum_{i=1}^{m} \int_{\Omega} g_{i} \mu_{i}$. If $F: \mathcal{Y} \rightarrow \mathbb{R} \cup\{+\infty\}$, then $F^{*}$ denotes its polar function $F^{*}\left(y^{*}\right) \equiv \sup \left\{\left\langle y^{*}, y\right\rangle-F(y) \mid y \in \mathcal{Y}\right\}$ and $\operatorname{dom} F \equiv\{y \in \mathcal{Y} \mid F(y)<\infty\}$ is the effective domain of $F$ (see [17]). If $Q$ is a subset of $\mathcal{Y}$, then $I_{Q}(\cdot)$ stands for its indicator function (taking the value 0 in $Q$ and $+\infty$ outside), and $I_{Q}^{*}(\cdot)$ stands for its support function.

Finally, we need the following notation. Let $V$ be a metric space. Then $B_{V}(\Xi, r)$ is the closed ball in $V$ with center $\Xi$ and radius $r$. Furthermore, $\operatorname{cl}_{V}(Z)$ stands for the closure of $Z$ (with $Z \subset V$ ) in the topology of the space $V$, while, analogously, $\operatorname{cl}_{\|\cdot\|}(Z)$ is the closure of the set $Z$ in the norm $\|\cdot\|$. Similarly, int $Z$ denotes the interior of $Z$. We will also consider the spaces $\mathbb{E}^{n}$ of real $n \times n$ matrices and $\mathbb{E}_{s}^{n}$ of symmetric real $n \times n$ matrices. In our paper we take $\left\|\left[e_{i j}\right]\right\|_{\mathbb{E}^{n}} \equiv \sum_{i, j=1}^{n}\left|e_{i j}\right|$ and $\|\cdot\|_{\mathbb{E}_{s}^{n}} \equiv\|\cdot\|_{\mathbb{E}^{n}}$, where $\left[e_{i j}\right] \in \mathbb{E}^{n}$. By $\otimes\left(\otimes_{s}\right.$ respectively) we denote the tensor product (symmetric tensor product, respectively). Let $\mathcal{L}^{0}\left(\Omega, \mathbb{R}^{m}\right)_{\mu}$ be the set of $\mu$-measurable 
functions from $\Omega$ into $\mathbb{R}^{m}$. If $\tau \subset 2^{X}$ is a linear topology in a vector space $X$, then $[X, \tau]$ denotes the topological space and $[X, \tau]^{*}$ the space dual to $[X, \tau]$. We define the following Banach spaces (see [24], [36], 37]):

$$
\begin{gathered}
L D(\Omega) \equiv\left\{\mathbf{u} \in L^{1}\left(\Omega, \mathbb{R}^{n}\right) \mid\right. \\
\left.\varepsilon_{i j}(\mathbf{u}) \equiv \frac{1}{2}\left(\frac{\partial u_{i}}{\partial x_{j}}+\frac{\partial u_{j}}{\partial x_{i}}\right) \in L^{1}(\Omega), i, j=1, \ldots, n\right\}, \\
B D(\Omega) \equiv\left\{\mathbf{u} \in L^{1}\left(\Omega, \mathbb{R}^{n}\right) \mid \varepsilon_{i j}(\mathbf{u}) \in \mathbb{M}_{b}(\Omega), i, j=1, \ldots, n\right\},
\end{gathered}
$$

with natural norms

$$
\|\mathbf{u}\|_{L D}=\|\mathbf{u}\|_{L^{1}}+\sum_{i, j}^{n}\left\|\varepsilon_{i j}(\mathbf{u})\right\|_{L^{1}},\|\mathbf{u}\|_{B D}=\|\mathbf{u}\|_{L^{1}}+\sum_{i, j}^{n}\left\|\varepsilon_{i j}(\mathbf{u})\right\|_{\mathbb{M}_{b}} .
$$

The space of rigid motions in $\mathbb{R}^{n}$ is denoted by $\mathcal{R}_{0} \equiv\{\mathbf{u} \in B D \mid \varepsilon(\mathbf{u})=\mathbf{0}\}$.

Proposition 1 (see [36]). Let $B D(\Omega)$ and $L^{1}\left(\operatorname{Fr} \Omega, \mathbb{R}^{n}\right)$ be endowed with the norm topologies. There exists a continuous surjective linear trace $\gamma_{B}$ of $B D(\Omega)$ into $L^{1}\left(\operatorname{Fr} \Omega, \mathbb{R}^{n}\right)$ such that $\boldsymbol{\gamma}_{B}(\mathbf{u})=\mathbf{u}_{\mid \operatorname{Fr}} \Omega$ for all $\mathbf{u} \in B D \cap$ $C\left(\bar{\Omega}, \mathbb{R}^{n}\right)$.

We define the spaces

$$
X \equiv C_{c}\left(\Omega, \mathbb{R}^{n}\right) \times C_{c}\left(\Omega, \mathbb{E}_{s}^{n}\right) \quad \text { and } \quad X_{0} \equiv\{(\mathbf{g}, \mathbf{h}) \in X \mid \mathbf{g}=\operatorname{div} \mathbf{h}\},
$$

endowed with the natural norm

$$
\|\mathbf{g}\|_{C\left(\Omega, \mathbb{R}^{n}\right)}+\|\mathbf{h}\|_{C\left(\Omega, \mathbb{E}_{s}^{n}\right)} \equiv \sup \left\{\|\mathbf{g}(x)\|_{\mathbb{R}^{n}} \mid x \in \Omega\right\}+\sup \left\{\|\mathbf{h}(x)\|_{\mathbb{E}_{s}^{n}} \mid x \in \Omega\right\}
$$

for every $\mathbf{g} \in C\left(\Omega, \mathbb{R}^{n}\right)$ and $\mathbf{h} \in C\left(\Omega, \mathbb{E}_{s}^{n}\right)$. Then $B D(\Omega)$ is isomorphic to the dual of $\left[\left(X / X_{0}\right),\|\cdot\|_{C\left(\Omega, \mathbb{R}^{n}\right)}+\|\cdot\|_{C\left(\Omega, \mathbb{E}_{s}^{n}\right)}\right]$ (see [36] and [37]).

The topology $\sigma\left(\left(X / X_{0}\right)^{*}, X\right)=\sigma\left(B D(\Omega), C_{c}\left(\Omega, \mathbb{R}^{n}\right) \times C_{c}\left(\Omega, \mathbb{E}_{s}^{n}\right)\right)$ is called the weak* $B D$ topology. A net $\left\{\mathbf{u}_{\delta}\right\}_{\delta \in D} \subset B D(\Omega)$ is convergent to $\mathbf{u}_{0} \in B D(\Omega)$ in the weak ${ }^{*} B D$ topology if and only if for all $(\mathbf{g}, \mathbf{h}) \in X$,

$$
\int_{\Omega} \mathbf{g} \cdot\left(\mathbf{u}_{0}-\mathbf{u}_{\delta}\right) d x+\int_{\Omega} \mathbf{h}: \boldsymbol{\varepsilon}\left(\mathbf{u}_{0}-\mathbf{u}_{\delta}\right) \rightarrow 0
$$

(see [18, pp. 73-81]). Nets are also called generalized sequences (cf. [15, pp. 26-29]). For every $\boldsymbol{\varphi} \in L^{1}\left(\operatorname{Fr} \Omega, \mathbb{R}^{n}\right)$, the set $\left\{\mathbf{u} \in B D(\Omega) \mid \boldsymbol{\gamma}_{B}(\mathbf{u})=\boldsymbol{\varphi}\right\}$ is dense in the space $[B D(\Omega)$, weak* topology] (see [5, Proposition 2.5]). If the space $L^{1}\left(\operatorname{Fr} \Omega, \mathbb{R}^{n}\right)$ is endowed with a Hausdorff topology, then the trace operator $\gamma_{B}$ is not continuous on $\left[B D(\Omega)\right.$, weak ${ }^{*}$ topology].

In this paper we define the Banach space of measurable functions

$$
W^{n}(\Omega, \operatorname{div}) \equiv\left\{\boldsymbol{\sigma} \in L^{\infty}\left(\Omega, \mathbb{E}_{s}^{n}\right) \mid \operatorname{div} \boldsymbol{\sigma} \in L^{n}\left(\Omega, \mathbb{R}^{n}\right)\right\}
$$


endowed with the natural norm

$$
\|\boldsymbol{\sigma}\|_{W^{n}(\Omega, \operatorname{div})}=\|\boldsymbol{\sigma}\|_{L^{\infty}\left(\Omega, \mathbb{E}_{s}^{n}\right)}+\|\operatorname{div} \boldsymbol{\sigma}\|_{L^{n}\left(\Omega, \mathbb{R}^{n}\right)}
$$

(cf. [36, Chapter 2, Section 7] and [5]). The distribution $\boldsymbol{\sigma}: \boldsymbol{\varepsilon}(\mathbf{u})$, where $\boldsymbol{\sigma} \in W^{n}(\Omega, \operatorname{div}), \mathbf{u} \in B D(\Omega)$, defined for every $\varphi_{1} \in C_{c}^{\infty}(\Omega)$ by

$$
\left\langle\boldsymbol{\sigma}: \boldsymbol{\varepsilon}(\mathbf{u}), \varphi_{1}\right\rangle_{D^{\prime} \times D}=-\int_{\Omega}(\operatorname{div} \boldsymbol{\sigma}) \cdot \mathbf{u} \varphi_{1} d x-\int_{\Omega} \boldsymbol{\sigma}:\left(\mathbf{u} \otimes \operatorname{grad} \varphi_{1}\right) d x,
$$

is a bounded measure on $\Omega$, and it is absolutely continuous with respect to $|\varepsilon(\mathbf{u})|$ (see [36]).

Assumption 1. Let $\Omega \subset \subset \Omega_{1}$ be bounded open connected sets of class $C^{1}$ in $\mathbb{R}^{n}$.

THEOREM 2 (cf. [36]). There exists a continuous, linear, surjective, open map $\boldsymbol{\beta}_{B}$ from $\left[W^{n}(\Omega, \operatorname{div}),\|\cdot\|_{W^{n}(\Omega, \text { div })}\right]$ onto $\left[L^{\infty}\left(\operatorname{Fr} \Omega, \mathbb{R}^{n}\right),\|\cdot\|_{L^{\infty}}\right]$ such that for every $\boldsymbol{\sigma} \in C\left(\bar{\Omega}, \mathbb{E}_{s}^{n}\right), \boldsymbol{\beta}_{B}(\boldsymbol{\sigma})=\left.\boldsymbol{\sigma}\right|_{\operatorname{Fr} \Omega} \cdot \boldsymbol{\nu}$, where $\boldsymbol{\nu}$ denotes the exterior unit vector normal to $\operatorname{Fr} \Omega$. Furthermore, for all $\mathbf{u} \in B D(\Omega)$ and all $\boldsymbol{\sigma} \in W^{n}(\Omega, \operatorname{div})$, the following Green formula holds:

$$
\int_{\Omega} \boldsymbol{\sigma}: \boldsymbol{\varepsilon}(\mathbf{u})+\int_{\Omega}(\operatorname{div} \boldsymbol{\sigma}) \cdot \mathbf{u} d x=\int_{\operatorname{Fr} \Omega} \boldsymbol{\beta}_{B}(\boldsymbol{\sigma}) \cdot \boldsymbol{\gamma}_{B}(\mathbf{u}) d s .
$$

In this paper, the Lebesgue and the Hausdorff measures on $\Omega$ and $\operatorname{Fr} \Omega$ are denoted by $d x$ and $d s$, respectively. Let $\Gamma_{0}$ and $\Gamma_{1}\left(\Gamma_{1}=\bar{\Gamma}_{1}\right)$ be Borel subsets of $\operatorname{Fr} \Omega$ such that $\Gamma_{0} \cap \Gamma_{1}=\emptyset$ and $d s\left(\operatorname{Fr} \Omega-\left(\Gamma_{0} \cup \Gamma_{1}\right)\right)=0$.

We will consider an elastic-perfectly plastic body, occupying the given set $\Omega$. Moreover, let $\mathcal{K}: \bar{\Omega} \rightarrow 2^{\mathbb{E}_{s}^{n}}$ be a multifunction such that the set $\mathcal{K}(x)$ denotes the elasticity convex domain at the point $x \in \Omega$.

Assumption 2 (cf. [5]). $\mathcal{K}(x)$ is a convex closed subset of $\mathbb{E}_{s}^{n}$ (for all $x \in \bar{\Omega})$ and the following conditions hold:

(i) if $\mathbf{z}(x) \in \mathcal{K}(x)$ for $d x$-almost every (dx-a.e.) $x \in \Omega, \mathbf{z} \in C\left(\bar{\Omega}, \mathbb{E}_{s}^{n}\right)$ and $\mathbf{z}_{\mid \operatorname{int} \Omega} \in W^{n}(\Omega, \operatorname{div})$, then $\mathbf{z}(y) \in \mathcal{K}(y)$ for every $y \in \bar{\Omega}$;

(ii) for every $y \in \bar{\Omega}$ and every $\mathbf{w} \in \mathcal{K}(y)$ there exists $\mathbf{z} \in C\left(\bar{\Omega}, \mathbb{E}_{s}^{n}\right)$ such that $\mathbf{z}_{\mid \text {int } \Omega} \in W^{n}(\Omega, \operatorname{div}), \mathbf{z}(y)=\mathbf{w}$ and $\mathbf{z}(x) \in \mathcal{K}(x)$ for every $x \in \bar{\Omega}$.

Conditions (i) and (ii) are equivalent to the condition that for every $y \in \bar{\Omega}$,

$$
\begin{aligned}
\mathcal{K}(y)=\left\{\mathbf{z}(y) \mid \mathbf{z} \in C\left(\bar{\Omega}, \mathbb{E}_{s}^{n}\right), \mathbf{z}_{\mid \text {int } \Omega}\right. & \in W^{n}(\Omega, \text { div }), \\
\mathbf{z}(x) & \in \mathcal{K}(x) \text { for } d x \text {-a.e. } x \in \Omega\} .
\end{aligned}
$$

Definition 1. A function $j^{*}: \Omega \times \mathbb{E}_{s}^{n} \rightarrow \mathbb{R} \cup\{+\infty\}$ is called a convex normal integrand if 
(i) the function $\mathbb{E}_{s}^{n} \ni \mathbf{w}^{*} \mapsto j^{*}\left(x, \mathbf{w}^{*}\right)$ is convex and l.s.c. for $d x$-a.e. $x \in \Omega$;

(ii) there exists a Borel function $\tilde{j^{*}}: \Omega \times \mathbb{E}_{s}^{n} \rightarrow \mathbb{R} \cup\{+\infty\}$ such that $\widetilde{j^{*}}(x, \cdot)=j^{*}(x, \cdot)$ for $d x$-a.e. $x \in \Omega$ (cf. [17, Chapter 8, p. 232]).

Moreover, let the following equality hold:

$$
\left\{\mathbf{w}^{*} \in \mathbb{E}_{s}^{n} \mid j^{*}\left(x, \mathbf{w}^{*}\right)<+\infty\right\}=\mathcal{K}(x) \text { for } d x \text {-a.e. } x \in \Omega .
$$

We define

$$
j(x, \mathbf{w}) \equiv j^{* *}(x, \mathbf{w}) \equiv \sup \left\{\mathbf{w}: \mathbf{w}^{*}-j^{*}\left(x, \mathbf{w}^{*}\right) \mid \mathbf{w}^{*} \in \mathbb{E}_{s}^{n}\right\}
$$

for $d x$-a.e. $x \in \Omega$ and for all $\mathbf{w} \in \mathbb{E}_{s}^{n}$. The function $j$ is a convex normal integrand (cf. [17, Chapter 8, Proposition 1.2]). Define $j_{\infty}: \bar{\Omega} \times \mathbb{E}_{s}^{n} \rightarrow$ $\mathbb{R} \cup\{+\infty\}$ by

$$
j_{\infty}(x, \mathbf{w}) \equiv \sup \left\{\mathbf{w}: \mathbf{w}^{*}-I_{\mathcal{K}(x)}\left(\mathbf{w}^{*}\right) \mid \mathbf{w}^{*} \in \mathbb{E}_{s}^{n}\right\}
$$

for all $x \in \bar{\Omega}$ and $\mathbf{w} \in \mathbb{E}_{s}^{n}$.

Let $\mathbf{f} \in L^{n}\left(\Omega, \mathbb{R}^{n}\right)$ and $\mathbf{g} \in L^{\infty}\left(\Gamma_{1}, \mathbb{R}^{n}\right)$. In this paper, we consider the following functional:

$$
B D(\Omega) \ni \mathbf{u} \mapsto F_{\lambda}(\mathbf{u})+G_{j}(\varepsilon(\mathbf{u})),
$$

where

$$
F_{\lambda}(\mathbf{u}) \equiv-\lambda L(\mathbf{u})+I_{C_{a}\left(\mathbf{u}^{0}\right)}(\mathbf{u}), \quad L(\mathbf{u}) \equiv \int_{\Omega} \mathbf{f} \cdot \mathbf{u} d x+\int_{\Gamma_{1}} \mathbf{g} \cdot \gamma_{B}(\mathbf{u}) d s,
$$

and kinematically admissible displacement fields $C_{a}\left(\mathbf{u}^{0}\right)$ are defined by

$$
C_{a}\left(\mathbf{u}^{0}\right) \equiv\left\{\mathbf{u} \in B D(\Omega) \mid \gamma_{B}(\mathbf{u})_{\mid \Gamma_{0}}=\mathbf{u}^{0} \text { on } \Gamma_{0}, \mathbf{u}^{0} \in L^{1}\left(\Gamma_{0}, \mathbb{R}^{n}\right)\right\} .
$$

The functional $G_{j}: \mathbb{M}_{b}\left(\Omega, \mathbb{E}_{s}^{n}\right) \rightarrow \mathbb{R} \cup\{+\infty\}$ is given by the expression

$$
G_{j}(\boldsymbol{\mu}) \equiv \begin{cases}\int_{\Omega} j(x, \boldsymbol{\mu}) d x & \text { if } \boldsymbol{\mu} \in L^{1}\left(\Omega, \mathbb{E}_{s}^{n}\right), \text { i.e. } \boldsymbol{\mu} \text { is absolutely } \\ +\infty & \text { continuous with respect to } d x \\ \text { otherwise. }\end{cases}
$$

The formula (3.14) describes the total elastic-perfectly plastic energy of a body occupying the given subset $\Omega$ of the space $\mathbb{R}^{n}$. This body is subjected to volume forces $\mathbf{f} \in L^{n}\left(\Omega, \mathbb{R}^{n}\right)$ and boundary forces $\mathbf{g} \in L^{\infty}\left(\Gamma_{1}, \mathbb{R}^{n}\right)$. The constant $0 \leq \lambda<+\infty$ is the load multiplier (see [36, Chapter 1, Sec. 4]). The body is clamped on $\Gamma_{0}$ (see [5] and [36]). The functional $(x, \boldsymbol{\varepsilon}(\mathbf{u})(x)) \mapsto j(x, \boldsymbol{\varepsilon}(\mathbf{u})(x))$ is the elastic-plastic potential, where $x \mapsto \mathbf{u}(x)$ is a displacement field. Moreover, $(x, \boldsymbol{\sigma}(x)) \mapsto j^{*}(x, \boldsymbol{\sigma}(x))$ is the dual elasticplastic potential, where $x \mapsto \boldsymbol{\sigma}(x)$ is a stress field. 
Let $\boldsymbol{\mu} \in \mathbb{M}_{b}\left(\Omega, \mathbb{E}_{s}^{n}\right)$. We recall that $|\boldsymbol{\mu}|$ is the total variation measure associated with $\boldsymbol{\mu}$, i.e. for every $\boldsymbol{\mu}$-measurable subset $\widetilde{\Omega}$ of $\Omega$,

$$
|\boldsymbol{\mu}|(\widetilde{\Omega})=\sup _{\boldsymbol{\varphi}}\left\{\int_{\widetilde{\Omega}} \boldsymbol{\varphi}: \boldsymbol{\mu} \mid \boldsymbol{\varphi} \in C\left(\bar{\Omega}, \mathbb{E}_{s}^{n}\right), \max _{i, j}\left\|\varphi_{i j}\right\|_{C(\Omega)} \leq 1\right\} .
$$

Then $\|\boldsymbol{\mu}\|_{\mathbb{M}_{b}(\Omega)}=\int_{\Omega}|\boldsymbol{\mu}|$. The density of $\boldsymbol{\mu}$ with respect to $|\boldsymbol{\mu}|$ will be denoted by $d \boldsymbol{\mu} / d|\boldsymbol{\mu}|$. Let $\boldsymbol{\mu}=\boldsymbol{\mu}_{a}(x) d x+\boldsymbol{\mu}_{s}$ be the Lebesgue decomposition of $\boldsymbol{\mu}$ into the absolutely continuous and singular parts with respect to $d x$.

\section{The scheme of duality and the extremal relation in Hencky} plasticity. We consider the duality between the displacement formulation (defined on $B D$ ) and the stress formulation of the variational problem in Hencky plasticity. We present the basic results of the paper at the end of this section. We consider the special case of the spaces $Y$ and $V$ below.

Let

$$
V \equiv B D(\Omega), \quad Y^{*} \equiv W^{n}(\Omega, \operatorname{div})
$$

and

$$
\begin{aligned}
Y= & Y(\Omega) \equiv \operatorname{span}\left(\varepsilon(B D(\Omega)), L^{1}\left(\Omega, \mathbb{E}_{s}^{n}\right)\right)=\left\{\boldsymbol{\mu} \in \mathbb{M}_{b}\left(\Omega, \mathbb{E}_{s}^{n}\right) \mid\right. \\
& \left.\exists \mathbf{u} \in B D(\Omega) \text { and } \exists \mathbf{w} \in L^{1}\left(\Omega, \mathbb{E}_{s}^{n}\right) \text { such that } \boldsymbol{\mu}=\boldsymbol{\varepsilon}(\mathbf{u})+\mathbf{w} d x\right\} .
\end{aligned}
$$

Definition 2. Let $\boldsymbol{\mu} \in Y$ and $\boldsymbol{\sigma} \in Y^{*}=W^{n}$ ( $\Omega$, div). Moreover, let $\boldsymbol{\mu}=\boldsymbol{\varepsilon}(\mathbf{u})+\mathbf{w} d x$, where $\mathbf{u} \in B D(\Omega)$ and $\mathbf{w} \in L^{1}\left(\Omega, \mathbb{E}_{s}^{n}\right)$. Then the measure $\boldsymbol{\sigma}: \boldsymbol{\mu}$ is defined by the formula $\boldsymbol{\sigma}: \boldsymbol{\mu}=\boldsymbol{\sigma}: \varepsilon(\mathbf{u})+\boldsymbol{\sigma}: \mathbf{w} d x$, where $\boldsymbol{\sigma}: \varepsilon(\mathbf{u})$ is given by (3.8).

Proposition 3. Let $\boldsymbol{\mu} \in Y$ and $\boldsymbol{\sigma} \in Y^{*}$. Moreover, let $\boldsymbol{\mu}=\boldsymbol{\varepsilon}(\mathbf{u})+$ $\mathbf{w} d x=\varepsilon\left(\mathbf{u}_{1}\right)+\mathbf{w}_{1} d x$, where $\mathbf{u}, \mathbf{u}_{1} \in B D(\Omega)$ and $\mathbf{w}, \mathbf{w}_{1} \in L^{1}\left(\Omega, \mathbb{E}_{s}^{n}\right)$. Then $\boldsymbol{\sigma}: \boldsymbol{\varepsilon}(\mathbf{u})+\boldsymbol{\sigma}: \mathbf{w} d x=\boldsymbol{\sigma}: \varepsilon\left(\mathbf{u}_{1}\right)+\boldsymbol{\sigma}: \mathbf{w}_{1} d x$ in the sense of equality of measures.

The space $W^{n}(\Omega, \operatorname{div})$ is endowed with the topology $\sigma\left(W^{n}(\Omega, \operatorname{div})\right.$, $Y(\Omega))$. Then $Y(\Omega)$ is dual to $W^{n}(\Omega$, div $)$, i.e., $\left[W^{n}(\Omega, \operatorname{div}), \sigma\left(W^{n}(\Omega, \operatorname{div})\right.\right.$, $Y(\Omega))]^{*}=Y(\Omega)$ (see [15, Theorem V.3.9]). We say that a sequence $\left\{\boldsymbol{\sigma}_{k}\right\}_{k \in \mathbb{N}}$ $\subset W^{n}(\Omega$, div $)$ converges to $\sigma_{0} \in W^{n}(\Omega, \operatorname{div})$ in the topology $\sigma\left(W^{n}(\Omega, \operatorname{div})\right.$, $Y(\Omega))$ if

$$
\int_{\Omega} \boldsymbol{\sigma}_{k}: \mathbf{w} \rightarrow \int_{\Omega} \boldsymbol{\sigma}_{0}: \mathbf{w}=\left\langle\mathbf{w}, \boldsymbol{\sigma}_{0}\right\rangle_{Y \times W^{n}(\Omega, \operatorname{div})}, \quad \forall \mathbf{w} \in Y(\Omega),
$$

as $k \rightarrow+\infty$. The space $Y(\Omega)$ is endowed with the topology $\sigma\left(Y(\Omega), W^{n}(\Omega\right.$, div)). We say that a sequence $\left\{\mathbf{w}_{k}\right\}_{k \in \mathbb{N}} \subset Y(\Omega)$ converges to $\mathbf{w}_{0} \in Y(\Omega)$ in $\sigma\left(Y(\Omega), W^{n}(\Omega, \operatorname{div})\right)$ if $\int_{\Omega} \boldsymbol{\sigma}: \mathbf{w}_{k} \rightarrow \int_{\Omega} \boldsymbol{\sigma}: \mathbf{w}_{0}$ for all $\left.\boldsymbol{\sigma} \in W^{n}(\Omega, \operatorname{div})\right)$. 
Let $V^{*}$ denote the space dual to $B D(\Omega)$ :

$$
V^{*}(\Omega)=V^{*} \equiv\left\{\left(\mathbf{u}^{*}, \varphi^{*}\right) \in L^{n}\left(\Omega, \mathbb{R}^{n}\right) \times L^{\infty}\left(\operatorname{Fr} \Omega, \mathbb{R}^{n}\right)\right\},
$$

where the duality between $B D(\Omega)$ and $V^{*}(\Omega)$ is given by

$$
\left\langle\mathbf{u},\left(\mathbf{u}^{*}, \varphi^{*}\right)\right\rangle_{B D \times V^{*}} \equiv \int_{\operatorname{Fr} \Omega} \boldsymbol{\varphi}^{*} \cdot \gamma_{B}(\mathbf{u}) d s-\int_{\Omega} \mathbf{u}^{*} \cdot \mathbf{u} d x .
$$

The space $B D(\Omega)$ is endowed with the topology $\sigma\left(B D(\Omega), V^{*}(\Omega)\right)$. Moreover, let $V^{*}(\Omega)$ be endowed with the topology $\sigma\left(V^{*}(\Omega), B D(\Omega)\right)$. The linear operator $\varepsilon:\left[B D, \sigma\left(B D, V^{*}\right)\right] \rightarrow\left[Y, \sigma\left(Y, W^{n}(\Omega, \operatorname{div})\right)\right]$ is continuous (see (3.9)). Then the explicit form of $\varepsilon^{*}: W^{n}(\Omega, \operatorname{div}) \rightarrow V^{*}$ is

$$
\begin{aligned}
\langle\varepsilon(\mathbf{u}), \boldsymbol{\sigma}\rangle_{Y \times Y^{*}} & =\int_{\operatorname{Fr} \Omega} \boldsymbol{\beta}_{B}(\boldsymbol{\sigma}) \boldsymbol{\gamma}_{B}(\mathbf{u}) d s-\int_{\Omega}(\operatorname{div} \boldsymbol{\sigma}) \mathbf{u} d x \\
& =\left\langle\mathbf{u}, \boldsymbol{\varepsilon}^{*}(\boldsymbol{\sigma})\right\rangle_{V \times V^{*}},
\end{aligned}
$$

i.e. $\boldsymbol{\varepsilon}^{*}(\boldsymbol{\sigma})=\left(\operatorname{div} \boldsymbol{\sigma}, \boldsymbol{\beta}_{B}(\boldsymbol{\sigma})\right)$.

In this paper we consider the original relaxed problem

$$
\left(R P_{\lambda, j}\right) \text { find } \inf \left\{F_{\lambda, R}(\mathbf{u})+G_{j}(\varepsilon(\mathbf{u})) \mid \mathbf{u} \in B D(\Omega)\right\},
$$

where $F_{\lambda, R}: B D(\Omega) \rightarrow \mathbb{R} \cup\{+\infty\}$ is defined by

$$
F_{\lambda, R}(\mathbf{u}) \equiv-\lambda\left(\int_{\Omega} \mathbf{f} \cdot \mathbf{u} d x+\int_{\Gamma_{1}} \mathbf{g} \cdot \gamma_{B}(\mathbf{u}) d s\right)+\int_{\Gamma_{0}} j_{\infty}\left(x,\left(\mathbf{u}^{0}-\gamma_{B}(\mathbf{u})\right) \otimes_{s} \boldsymbol{\nu}\right) d s
$$

Here, $j_{\infty}$ is the relaxation of the Dirichlet boundary condition on $\Gamma_{0}$. The elastic-plastic potential $G_{j}$ is given by the formula (3.17).

Proposition 4. The dual potential $G_{j}^{*}: W^{n}(\Omega, \operatorname{div}) \rightarrow \mathbb{R} \cup\{+\infty\}$ defined by

$$
G_{j}^{*}(\boldsymbol{\sigma}) \equiv \sup \left\{\int_{\Omega} \boldsymbol{\sigma}: \boldsymbol{\mu}-G_{j}(\boldsymbol{\mu}) \mid \boldsymbol{\mu} \in Y(\Omega)\right\}
$$

satisfies $G_{j}^{*}(\boldsymbol{\sigma})=\int_{\Omega} j^{*}(x, \boldsymbol{\sigma}) d x$ for every $\boldsymbol{\sigma} \in W^{n}(\Omega, \operatorname{div})$.

Proof. By (3.17) and [34, Theorem 3A and Proposition 2M], we have

$$
G_{j}^{*}(\boldsymbol{\sigma}) \equiv \sup \left\{\int_{\Omega}[\boldsymbol{\sigma}: \mathbf{w}-j(x, \mathbf{w})] d x \mid \mathbf{w} \in L^{1}\right\}=\int_{\Omega} j^{*}(x, \boldsymbol{\sigma}) d x .
$$

Definition 3. Define the bidual potential $G_{j}^{* *}: Y(\Omega) \rightarrow \mathbb{R} \cup\{+\infty\}$ by

$$
G_{j}^{* *}(\mathbf{w}) \equiv \sup \left\{\int_{\Omega} \boldsymbol{\sigma}: \mathbf{w}-G_{j}^{*}(\boldsymbol{\sigma}) \mid \boldsymbol{\sigma} \in W^{n}(\Omega, \operatorname{div})\right\}
$$

for every $\mathbf{w} \in Y(\Omega)$. 
TheOrem 5. The bidual potential $G_{j}^{* *}$ satisfies

$$
G_{j}^{* *}(\varepsilon(\mathbf{u}))=\int_{\Omega} j\left(x, \varepsilon(\mathbf{u})_{a}\right) d x+\int_{\Omega} j_{\infty}\left(x, \frac{d\left(\varepsilon(\mathbf{u})_{s}\right)}{d\left|\varepsilon(\mathbf{u})_{s}\right|}\right) d\left|\varepsilon(\mathbf{u})_{s}\right|
$$

for every $\mathbf{u} \in B D(\Omega)$, where $\boldsymbol{\varepsilon}(\mathbf{u})_{a}$ is the absolutely continuous part and $\varepsilon(\mathbf{u})_{s}$ the singular part of $\varepsilon(\mathbf{u})$ with respect to $d x$.

The component $\int_{\Omega} j_{\infty}\left(d\left(\varepsilon(\mathbf{u})_{s}\right) / d\left|\varepsilon(\mathbf{u})_{s}\right|\right) d\left|\varepsilon(\mathbf{u})_{s}\right|$ characterizes discontinuities of the field of displacements.

Proposition 6. Let $F_{\lambda, R}$ be given by (4.8); then the dual functional $\left(F_{\lambda, R}\right)^{*}: V^{*}(\Omega) \rightarrow \mathbb{R} \cup\{+\infty\}$, defined by

$$
\left(F_{\lambda, R}\right)^{*}\left(-\varepsilon^{*}(\boldsymbol{\sigma})\right) \equiv \sup \left\{\left\langle\mathbf{u},-\varepsilon^{*}(\boldsymbol{\sigma})\right\rangle_{B D \times V^{*}}-F_{\lambda, R}(\mathbf{u}) \mid \mathbf{u} \in B D(\Omega)\right\},
$$

satisfies

$$
\left(F_{\lambda, R}\right)^{*}\left(-\varepsilon^{*}(\boldsymbol{\sigma})\right)= \begin{cases}-\int_{\Gamma_{0}} \boldsymbol{\beta}_{B}(\boldsymbol{\sigma}) \cdot \mathbf{u}^{0} d s \quad & \text { if } \operatorname{div} \boldsymbol{\sigma}=-\lambda \mathbf{f} \text { in } \Omega \\ & \boldsymbol{\beta}_{B}(\boldsymbol{\sigma})=\lambda \mathbf{g} \text { on } \Gamma_{1} \text { and } \\ & \boldsymbol{\beta}_{B}(\boldsymbol{\sigma})(x) \in \mathcal{K}(x) \cdot \boldsymbol{\nu}(x) \\ & \text { for ds-a.e. } x \in \Gamma_{0}, \\ & \text { otherwise }\end{cases}
$$

for every $\boldsymbol{\sigma} \in W^{n}(\Omega$, div) (cf. (4.5), (4.6)).

In the above case $-\int_{\Gamma_{0}} \boldsymbol{\beta}_{B}(\boldsymbol{\sigma}) \cdot \mathbf{u}^{0} d s$ is the work on $\Gamma_{0}$, where $\mathbf{u}^{0}$ is the displacement field forced on the boundary $\Gamma_{0}$.

Proposition 7. The bidual functional $\left(F_{\lambda, R}\right)^{* *}: B D(\Omega) \rightarrow \mathbb{R} \cup\{+\infty\}$ defined by

$$
\left(F_{\lambda, R}\right)^{* *}(\mathbf{u}) \equiv \sup \left\{\left\langle\mathbf{u},\left(\mathbf{u}^{*}, \boldsymbol{\varphi}^{*}\right)\right\rangle_{B D \times V^{*}}-\left(F_{\lambda, R}\right)^{*}\left(\mathbf{u}^{*}, \boldsymbol{\varphi}^{*}\right) \mid\left(\mathbf{u}^{*}, \boldsymbol{\varphi}^{*}\right) \in V^{*}\right\},
$$

where $\left(F_{\lambda, R}\right)^{*}\left(\mathbf{u}^{*}, \boldsymbol{\varphi}^{*}\right) \equiv \sup \left\{\left\langle\mathbf{u},\left(\mathbf{u}^{*}, \boldsymbol{\varphi}^{*}\right)\right\rangle_{B D \times V^{*}}-F_{\lambda, R}(\mathbf{u}) \mid \mathbf{u} \in B D(\Omega)\right\}$, satisfies the equality $\left(F_{\lambda, R}\right)^{* *}(\mathbf{u})=F_{\lambda, R}(\mathbf{u})$ for every $\mathbf{u} \in B D(\Omega)$ (cf. (4.8)).

THEOREM 8. The dual relaxed problem $\left(R P_{\lambda, j}^{*}\right)$ is

$$
\text { find } \sup \left\{-\left(F_{\lambda, R}\right)^{*}\left(-\varepsilon^{*}(\boldsymbol{\sigma})\right)-G_{j}^{*}(\boldsymbol{\sigma}) \mid \boldsymbol{\sigma} \in W^{n}(\Omega, \operatorname{div})\right\}
$$

(cf. Propositions 4, 6 and Section 2).

THEOREM 9. The bidual relaxed problem $\left(R P_{\lambda, j}^{* *}\right)$ is

$$
\text { find } \inf \left\{\left(F_{\lambda, R}\right)^{* *}(\mathbf{u})+G_{j}^{* *}(\varepsilon(\mathbf{u})) \mid \mathbf{u} \in B D(\Omega)\right\}
$$

(cf. Theorem 5, Proposition 7, formula (4.8) and Section 2).

A maximizer of $\left(R P_{\lambda, j}^{*}\right)$ is a solution of the stress problem. Similarly, a minimizer of $\left(R P_{\lambda, j}^{* *}\right)$ is a solution of the displacement problem.

Due to [17, Chapter 3] we have $\left(R P_{\lambda, j}^{* * *}\right)=\left(R P_{\lambda, j}^{*}\right)$. 
5. Additional assumptions and supplementary information. In this section we state the remaining mathematical assumptions. Moreover, we formulate the essential definitions and properties of the spaces considered. We begin by formulating Assumptions 3 and 4 .

Assumption 3. There exist $k>0$ and $r_{1}>0$ such that

$$
0 \leq j^{*}\left(x, \mathbf{w}^{*}\right) \leq k, \quad \forall \mathbf{w}^{*} \in B_{\mathbb{E}_{s}^{n}}\left(0, r_{1}\right), \quad \text { for } d x \text {-a.e. } x \in \Omega,
$$

and $j^{*}$ is nonnegative on $\Omega \times \mathbb{E}_{s}^{n}$. Moreover, for every $\widehat{r}>0$ there exists $c_{\widehat{r}}$ such that

$$
\begin{aligned}
& \sup \left\{\int_{\Omega} j^{*}\left(x, \mathbf{z}^{*}\right) d x\right. \mathbf{z}^{*} \in L^{\infty}\left(\Omega, \mathbb{E}_{s}^{n}\right),\left\|\mathbf{z}^{*}\right\|_{L^{\infty}}<\widehat{r} \\
&\text { and } \left.\mathbf{z}^{*}(x) \in \mathcal{K}(x) \text { for } d x \text {-a.e. } x \in \Omega\right\}<c_{\widehat{r}}<+\infty .
\end{aligned}
$$

That is, the dual elastic potential $\mathbf{z}^{*} \mapsto \int_{\Omega} j^{*}\left(x, \mathbf{z}^{*}\right) d x$ is finite for every $\mathbf{z}^{*} \in L^{\infty}\left(\Omega, \mathbb{E}_{s}^{n}\right)$ such that $\mathbf{z}^{*}$ is an admissible stress field. From (3.11) and (5.1) we get $B_{\mathbb{E}_{s}^{n}}\left(0, r_{1}\right) \subset \mathcal{K}(x)$ for $d x$-a.e. $x \in \Omega$.

Definition 4 (see [20]). A Borel set $\mathcal{C} \subseteq \mathbb{R}^{n}$ is called a Caccioppoli set if

$$
\sup \left\{\int_{\mathcal{C}}(\operatorname{div} \widetilde{f}) d x \mid \tilde{f} \in C_{0}^{1}\left(\Omega_{2}, \mathbb{R}^{n}\right),\|\widetilde{f}(x)\|_{\mathbb{R}^{n}} \leq 1 \forall x \in \Omega_{2}\right\}<+\infty
$$

for all bounded open subsets $\Omega_{2}$ of $\mathbb{R}^{n}$.

That is, the Hausdorff measure of the set $\operatorname{Fr} \mathcal{C}$ is finite (cf. (3.9)).

Assumption 4. Let $\Gamma_{1}=\operatorname{Fr} \Omega \cap \mathcal{C}$, where $\mathcal{C}=\operatorname{clint} \mathcal{C} \subset \Omega_{1}$ is a closed Caccioppoli set and $d s(\operatorname{Fr} \Omega \cap \operatorname{Fr} \mathcal{C})=0$.

Proposition 10 (see [36, p. 255]). If $\mathbf{u} \in B D\left(\Omega_{1}\right)$, then

$$
\varepsilon(\mathbf{u})=\varepsilon(\mathbf{u})_{\mid \Omega}+\left.\varepsilon(\mathbf{u})\right|_{\Omega_{1}-\bar{\Omega}}+\left(\gamma_{B}^{O}(\mathbf{u})-\gamma_{B}^{I}(\mathbf{u})\right) \otimes_{s} \boldsymbol{\nu} d s,
$$

where the inner trace $\boldsymbol{\gamma}_{B}^{I}: B D(\Omega) \rightarrow L^{1}\left(\operatorname{Fr} \Omega, \mathbb{R}^{n}\right)$ and outer trace $\boldsymbol{\gamma}_{B}^{O}$ : $B D\left(\Omega_{1}-\bar{\Omega}\right) \rightarrow L^{1}\left(\operatorname{Fr} \Omega, \mathbb{R}^{n}\right)$ are given by the formulae $\gamma_{B}^{I}(\mathbf{u})=\mathbf{u}_{\mid \operatorname{Fr}} \Omega$ for $\mathbf{u} \in B D(\Omega) \cap C\left(\bar{\Omega}, \mathbb{R}^{n}\right)$, and $\boldsymbol{\gamma}_{B}^{O}(\mathbf{u})=\mathbf{u}_{\mid \operatorname{Fr} \Omega}$ for $\mathbf{u} \in B D\left(\Omega_{1}-\bar{\Omega}\right) \cap$ $C\left(\Omega_{1}-\Omega, \mathbb{R}^{n}\right)$, respectively, and where $\otimes_{s}$ denotes the symmetric tensor product: $\left(\mathbf{p} \otimes_{s} \boldsymbol{\nu}\right)_{i j} \equiv\left(p_{i} \nu_{j}+p_{j} \nu_{i}\right) / 2$.

Lemma 11 (cf. [8, Lemma 5], [5, Lemma 4.18] and [2, Theorem 3.1]). If there exists a closed Caccioppoli set $\mathcal{C} \subset \Omega_{1}(\mathcal{C}=\operatorname{clint} \mathcal{C})$ such that $\Gamma_{2}=\operatorname{Fr} \Omega \cap \mathcal{C}$, with $d s(\operatorname{Fr} \Omega \cap \operatorname{Fr} \mathcal{C})=0$, then for all $\mathbf{u} \in B D\left(\Omega_{1}\right)$ and all $\boldsymbol{\sigma} \in W^{n}\left(\Omega_{1}\right.$, div $)$,

$$
\int_{\Gamma_{2}} \boldsymbol{\beta}_{B}\left(\boldsymbol{\sigma}_{\mid \Omega}\right) \cdot\left(\boldsymbol{\gamma}_{B}^{O}(\mathbf{u})-\gamma_{B}^{I}(\mathbf{u})\right) d s=\int_{\Gamma_{2}} \boldsymbol{\sigma}:\left[\left(\boldsymbol{\gamma}_{B}^{O}(\mathbf{u})-\gamma_{B}^{I}(\mathbf{u})\right) \otimes_{s} \boldsymbol{\nu}\right] d s,
$$

where we denote $\boldsymbol{\sigma}: \boldsymbol{\varepsilon}(\mathbf{u})_{\mid \operatorname{Fr} \Omega}$ by $\boldsymbol{\sigma}:\left[\left(\boldsymbol{\gamma}_{B}^{O}(\mathbf{u})-\gamma_{B}^{I}(\mathbf{u})\right) \otimes_{s} \boldsymbol{\nu}\right] d s$. 
REMARK 1 (see [5, Lemma 2.13]). For each $\boldsymbol{\sigma} \in W^{n}(\Omega$, div) there exists $\boldsymbol{\sigma}_{1} \in W^{n}\left(\Omega_{1}\right.$, div $)$ such that $\boldsymbol{\sigma}_{1 \mid \Omega}=\boldsymbol{\sigma}$.

It is easy to obtain the following inequalities. By the formula (5.1), we have

$$
c_{n} r_{1}\|\mathbf{w}\|_{\mathbb{E}_{s}^{n}}-k \leq j(x, \mathbf{w}) \quad \text { for } d x \text {-a.e. } x \in \Omega,
$$

where the positive constant $c_{n}$ depends only on the dimension of $\mathbb{E}_{s}^{n}$. By Appendix A and [17. Chapter 8, Proposition 1.2], $j_{\infty}$ is a convex normal integrand. Because of (3.11) and (5.1), we have, for all $x \in \bar{\Omega}$,

$$
c_{n} r_{1}\|\mathbf{w}\|_{\mathbb{E}_{s}^{n}} \leq j_{\infty}(x, \mathbf{w}) .
$$

The following example confirms the fact that the multifunction $\mathcal{K}$ which satisfies Assumption 2 describes a composite material with a fluctuating plastic yield condition.

EXAMPLE. We define a multifunction $\mathcal{K}_{A}$ on $\Omega_{A}=B_{\mathbb{R}^{n}}\left(0, R_{A}\right)$, valued in $\mathbb{E}_{s}^{n}$, by

$$
\mathcal{K}_{A}\left(x_{1}, \ldots, x_{n}\right)= \begin{cases}B_{\mathbb{E}_{s}^{n}}\left(0, R_{2}\right) & \text { if } x_{1} \leq 0, \\ B_{\mathbb{E}_{s}^{n}}\left(0, R_{3}\right) & \text { if } x_{1}>0,\end{cases}
$$

where $0<R_{2}<R_{3}<+\infty$. We show that $\mathcal{K}_{A}$ satisfies Assumption 2 , where $\mathcal{K}$ is replaced by $\mathcal{K}_{A}$ and $\Omega$ is replaced by $\Omega_{A}$. Indeed, for every $\delta>0$ there exists a smooth function $h_{\delta}: \mathbb{R} \rightarrow\left[1, R_{3} / R_{2}\right]$ such that $h_{\delta}(x)=1$ for $x \leq 0$, $h_{\delta}(x)=R_{3} / R_{2}$ for $x \geq \delta$ and $h_{\delta}(x) \in\left[1, R_{3} / R_{2}\right]$ for $x \in(0, \delta)$. Then $\mathcal{K}_{A}\left(x_{1}, \ldots, x_{n}\right)=\left\{\left(y_{1}, \ldots, y_{n^{2}}\right) h_{\delta}\left(x_{1}\right) \mid\left(y_{1}, \ldots, y_{n^{2}}\right) \in B_{\mathbb{E}_{s}^{n}}\left(0, R_{2}\right), \delta \in(0,1)\right\}$.

Definition 5 (see [36] and [18, Chapter 1, Sec. 6]). A net $\left\{\mathbf{u}_{\delta}\right\}_{\delta \in D} \subset$ $B D(\Omega)$ converges to $\mathbf{u}_{0}$ (in the topology (5.8)-(5.9)) if and only if

$$
\begin{aligned}
& \mathbf{u}_{\delta} \rightarrow \mathbf{u}_{0} \quad \text { in }\|\cdot\|_{L^{p}\left(\Omega, \mathbb{R}^{n}\right)} \text { for each } p \text { such that } 1 \leq p<q=n /(n-1) \\
& \text { and weakly in } L^{q}\left(\Omega, \mathbb{R}^{n}\right) ; \text { if } n=1 \text { then } q=\infty, \\
& \boldsymbol{\varepsilon}\left(\mathbf{u}_{\delta}\right) \rightarrow \boldsymbol{\varepsilon}\left(\mathbf{u}_{0}\right) \text { weak }^{*} \text { in } \mathbb{M}_{b}\left(\Omega, \mathbb{E}_{s}^{n}\right) .
\end{aligned}
$$

Proposition 12 (cf. [5] and [8, Proposition 2]). The weak $B D(\Omega)$ topology and the topology (5.8)-(5.9) are equivalent on bounded subsets of $B D(\Omega)$.

The injection of $\left[B D(\Omega)\right.$, weak $\left.{ }^{*}\right]$ into $\left[L^{p}\left(\Omega, \mathbb{R}^{n}\right)\right.$, weak topology $]$ is continuous on bounded subsets of $B D(\Omega)$, where $1 \leq p \leq q=n /(n-1)$; if $n=1$ then $q=\infty$.

For completeness we provide the following proof:

Proof of Proposition 3. By [36, Chapter 2, Lemma 7.1] there exists a sequence $\left\{\boldsymbol{\sigma}_{m}\right\}_{m \in \mathbb{N}} \subset C^{\infty}\left(\bar{\Omega}, \mathbb{E}_{s}^{n}\right)$ such that

$$
\boldsymbol{\sigma}_{m} \rightarrow \boldsymbol{\sigma} \text { in }\|\cdot\|_{L^{p}\left(\Omega, \mathbb{E}_{s}^{n}\right)}(n<p<\infty) \text { and } \operatorname{div} \boldsymbol{\sigma}_{m} \rightarrow \operatorname{div} \boldsymbol{\sigma} \text { in }\|\cdot\|_{L^{n}}
$$


By (3.8) we have

$$
\begin{aligned}
& \int_{\Omega}\left[\left(\operatorname{div} \boldsymbol{\sigma}_{m}\right) \cdot \mathbf{u} \varphi+\boldsymbol{\sigma}_{m}:(\mathbf{u} \otimes \nabla \varphi)\right] d x=\int_{\Omega}\left(\operatorname{div} \boldsymbol{\sigma}_{m}\right) \cdot\left(\mathbf{u}-\mathbf{u}_{1}\right) \varphi d x \\
+ & \int_{\Omega} \boldsymbol{\sigma}_{m}:\left(\left(\mathbf{u}-\mathbf{u}_{1}\right) \otimes \nabla \varphi\right) d x+\int_{\Omega}\left[\left(\operatorname{div} \boldsymbol{\sigma}_{m}\right) \cdot \mathbf{u}_{1} \varphi+\boldsymbol{\sigma}_{m}:\left(\mathbf{u}_{1} \otimes \nabla \varphi\right)\right] d x
\end{aligned}
$$

for every $\varphi \in C_{0}^{\infty}(\Omega)$. Since $\mathbf{u}, \mathbf{u}_{1} \in B D(\Omega)$, we have $\mathbf{u}, \mathbf{u}_{1} \in L^{n /(n-1)}\left(\Omega, \mathbb{R}^{n}\right)$ (see [36, Chapter 2, Theorem 2.2]). Therefore, (5.11) holds with $\boldsymbol{\sigma}_{m}$ replaced by $\boldsymbol{\sigma}$ (cf. (3.8)), and we obtain the conclusion.

6. The auxiliary scheme of duality. In this section we define an auxiliary duality between the displacement formulation and the stress formulation of the variational problem in Hencky plasticity (cf. Section 2). We prove, similarly to [38], the existence theorem for the stress problem (see Theorem 14 for an elastic-perfectly plastic solid, made of a non-homogeneous Hencky material) where the following condition is fulfilled:

$$
\exists r_{2}>0, \forall x \in \bar{\Omega} \quad \mathcal{K}(x) \subset B_{\mathbb{E}_{s}^{n}}\left(0, r_{2}\right) .
$$

Let

$$
\widetilde{V} \equiv\left[L D(\Omega),\|\cdot\|_{L D}\right], \quad \widetilde{Y} \equiv\left[L^{1}\left(\Omega, \mathbb{E}_{s}^{n}\right),\|\cdot\|_{L^{1}\left(\Omega, \mathbb{E}_{s}^{n}\right)}\right]
$$

(cf. Section 2). Moreover, let

$$
\widetilde{V}^{*}=L D^{*}(\Omega)=\left[L D(\Omega),\|\cdot\|_{L D}\right]^{*}, \widetilde{Y}^{*}=\left[L^{\infty}\left(\Omega, \mathbb{E}_{s}^{n}\right), \sigma\left(L^{\infty}, L^{1}\right)\right] .
$$

The linear operator $\varepsilon: L D(\Omega) \rightarrow L^{1}\left(\Omega, \mathbb{E}_{s}^{n}\right)=\widetilde{Y}$ is continuous (cf. (3.1)). Below, the following functional is considered:

$$
L D(\Omega) \ni \mathbf{u} \mapsto F_{\lambda, 1}(\mathbf{u})+G_{1, j}(\varepsilon(\mathbf{u})),
$$

where $F_{\lambda, 1}(\mathbf{u})=F_{\lambda}(\mathbf{u})$ for every $\mathbf{u} \in L D(\Omega)$ and $G_{1, j}(\varepsilon(\mathbf{u}))=G_{j}(\varepsilon(\mathbf{u}))$ for every $\mathbf{u} \in L D(\Omega)$ (cf. (3.15) and (3.17)). Let $\gamma_{B}\left(\mathbf{u}_{0}\right)=\mathbf{u}^{0}$ on $\Gamma_{0}$ where $\mathbf{u}_{0} \in L D(\Omega)$ (see [36, Chapter 2, Theorem 1.1]).

Lemma 13 (cf. [38] and [36, Chapter 1, Lemma 2.2]). The dual problem to

$$
\left(P_{\lambda, j}\right) \quad \text { find } \inf \left\{F_{\lambda, 1}(\mathbf{u})+G_{1, j}(\varepsilon(\mathbf{u})) \mid \mathbf{u} \in L D(\Omega)\right\}
$$

$i s$

(6.6) $\left(P_{\lambda, j}^{*}\right) \quad$ find $\sup \left\{-\left(F_{\lambda, 1}\right)^{*}\left(-\varepsilon^{*}(\boldsymbol{\sigma})\right)-G_{1, j}^{*}(\boldsymbol{\sigma}) \mid \boldsymbol{\sigma} \in L^{\infty}\left(\Omega, \mathbb{E}_{s}^{n}\right)\right\}$, 
where

$$
\begin{aligned}
\left(F_{\lambda, 1}\right)^{*}\left(-\varepsilon^{*}(\boldsymbol{\sigma})\right) & = \begin{cases}-\int_{\Gamma_{0}} \boldsymbol{\beta}_{B}(\boldsymbol{\sigma}) \cdot \mathbf{u}^{0} d s & \text { if } \operatorname{div} \boldsymbol{\sigma}=-\lambda \mathbf{f} \text { in } \Omega, \\
+\infty & \text { and } \boldsymbol{\beta}_{B}(\boldsymbol{\sigma})=\lambda \mathbf{g} \text { on } \Gamma_{1},\end{cases} \\
G_{1, j}^{*}(\boldsymbol{\sigma}) & =\int_{\Omega} j^{*}(x, \boldsymbol{\sigma}) d x .
\end{aligned}
$$

The trace $\boldsymbol{\beta}_{B}(\boldsymbol{\sigma})$ on $\operatorname{Fr} \Omega$ exists, since $\operatorname{div} \boldsymbol{\sigma}=-\lambda \mathbf{f} \in L^{n}\left(\Omega, \mathbb{R}^{n}\right)$ (cf. Theorem 2).

Proof. (i) First we prove (6.8). Because of (3.12), the function $j$ is a convex normal integrand. By [34, Theorem 3A and Proposition 2M] we get (6.8), since $L^{1}\left(\Omega, \mathbb{E}_{s}^{n}\right)$ is a decomposable space and $j(x, \mathbf{0}) \leq 0$ for $d x$-a.e. $x \in \Omega\left(j^{*}\right.$ is a nonnegative function).

(ii) We apply Lemma 2.1 of [36, Chapter 1] with $v_{0}=\mathbf{u}_{0} \in \widetilde{V}=L D(\Omega)$,

$$
\left\langle v_{0}^{*}, \mathbf{u}\right\rangle_{L D^{*} \times L D}=-\lambda\left(\int_{\Omega} \mathbf{f} \cdot \mathbf{u} d x+\int_{\Gamma_{1}} \mathbf{g} \cdot \gamma_{B}(\mathbf{u}) d s\right), \quad \forall \mathbf{u} \in L D(\Omega),
$$

and where $\mathcal{B}$ is the set of $\mathbf{u}$ in $L D(\Omega)$ such that $\gamma_{B}(\mathbf{u})$ vanishes on $\Gamma_{0}$. We deduce from the lemma that $\left(F_{\lambda, 1}\right)^{*}\left(-\boldsymbol{\varepsilon}^{*}(\boldsymbol{\sigma})\right)$ is equal to

$$
Q_{\mathbf{u}_{0}}(\boldsymbol{\sigma}) \equiv\left\langle-\varepsilon^{*}(\boldsymbol{\sigma}), \mathbf{u}_{0}\right\rangle_{L D^{*} \times L D}+\lambda\left(\int_{\Omega} \mathbf{f} \cdot \mathbf{u}_{0} d x+\int_{\Gamma_{1}} \mathbf{g} \cdot \gamma_{B}\left(\mathbf{u}_{0}\right) d s\right)
$$

if $Q_{\mathbf{u}}(\boldsymbol{\sigma})=0$ for every $\mathbf{u} \in \mathcal{B}$, and to $+\infty$ if $Q_{\mathbf{u}_{1}}(\boldsymbol{\sigma}) \neq 0$ for some $\mathbf{u}_{1} \in \mathcal{B}$. Below we consider $\boldsymbol{\sigma} \in L^{\infty}\left(\Omega, \mathbb{E}_{s}^{n}\right)$ such that $Q_{\mathbf{u}}(\boldsymbol{\sigma})=0$ for every $\mathbf{u} \in \mathcal{B}$. In $Q_{\mathbf{u}}(\boldsymbol{\sigma})$ we replace $\mathbf{u}$ by $\widehat{\mathbf{u}} \in L D_{0} \equiv\left\{\mathbf{u} \in L D(\Omega) \mid \gamma_{B}(\mathbf{u})=\mathbf{0}\right.$ on Fr $\left.\Omega\right\}$; then

$$
\begin{aligned}
\langle-\boldsymbol{\sigma}, \boldsymbol{\varepsilon}(\widehat{\mathbf{u}})\rangle_{L^{\infty} \times L^{1}}+\lambda \int_{\Omega} \mathbf{f} \cdot \widehat{\mathbf{u}} d x & =0, \quad \forall \widehat{\mathbf{u}} \in L D_{0}(\Omega), \\
\left\langle-\boldsymbol{\varepsilon}^{*}(\boldsymbol{\sigma}), \widehat{\mathbf{u}}\right\rangle_{L D^{*} \times L D}+\lambda \int_{\Omega} \mathbf{f} \cdot \widehat{\mathbf{u}} d x & =\left\langle\lambda \mathbf{f}-\boldsymbol{\varepsilon}^{*}(\boldsymbol{\sigma}), \widehat{\mathbf{u}}\right\rangle_{L D^{*} \times L D}=0
\end{aligned}
$$

for every $\widehat{\mathbf{u}} \in L D_{0}(\Omega)$. By (3.9) and (6.11), $\int_{\Omega}(\lambda \mathbf{f}+\operatorname{div} \boldsymbol{\sigma}) \cdot \widehat{\mathbf{u}} d x=0$ for every $\widehat{\mathbf{u}} \in C_{0}^{\infty}\left(\Omega, \mathbb{R}^{n}\right)$ (cf. [5, formula (4.17)]) or in other words $\lambda \mathbf{f}=-\operatorname{div} \boldsymbol{\sigma}$ in the sense of distributions on $\Omega$. The trace $\boldsymbol{\beta}_{B}(\boldsymbol{\sigma})$ on $\operatorname{Fr} \Omega$ exists, because $\operatorname{div}(\boldsymbol{\sigma})=-\lambda \mathbf{f} \in L^{n}\left(\Omega, \mathbb{R}^{n}\right)$ (see Theorem 2 ). By the equality $Q_{\mathbf{u}}(\boldsymbol{\sigma})=0$ for every $\mathbf{u} \in \mathcal{B}$, and by (3.9), we have

$$
0=\int_{\Omega}(\lambda \mathbf{f}+\operatorname{div} \boldsymbol{\sigma}) \cdot \mathbf{u} d x+\int_{\Gamma_{1}}\left(\lambda \mathbf{g}-\boldsymbol{\beta}_{B}(\boldsymbol{\sigma})\right) \cdot \gamma_{B}(\mathbf{u}) d s, \quad \forall \mathbf{u} \in \mathcal{B},
$$


because for every $\mathbf{u} \in \mathcal{B}, \gamma_{B}(\mathbf{u})=\mathbf{0}$ on $\Gamma_{0}$. Therefore,

$$
0=-\int_{\Gamma_{1}} \boldsymbol{\beta}_{B}(\boldsymbol{\sigma}) \cdot \boldsymbol{\gamma}_{B}(\mathbf{u}) d s+\lambda \int_{\Gamma_{1}} \mathbf{g} \cdot \boldsymbol{\gamma}_{B}(\mathbf{u}) d s, \quad \forall \mathbf{u} \in \mathcal{B},
$$

since $\operatorname{div} \boldsymbol{\sigma}=-\lambda \mathbf{f}$. The trace $\boldsymbol{\gamma}_{B}$ is a function onto $L^{1}\left(\operatorname{Fr} \Omega, \mathbb{R}^{n}\right)$, so $\boldsymbol{\beta}_{B}(\boldsymbol{\sigma})=$ $\lambda \mathbf{g}$ on $\Gamma_{1}$. By (6.10) and (3.9) we obtain

$$
\begin{aligned}
& \left(F_{\lambda, 1}\right)^{*}\left(-\varepsilon^{*}(\boldsymbol{\sigma})\right) \\
= & \left\langle-\boldsymbol{\sigma}, \boldsymbol{\varepsilon}\left(\mathbf{u}_{0}\right)\right\rangle_{L^{\infty} \times L^{1}}+\lambda\left(\int_{\Omega} \mathbf{f} \cdot \mathbf{u}_{0} d x+\int_{\Gamma_{1}} \mathbf{g} \cdot \boldsymbol{\gamma}_{B}\left(\mathbf{u}_{0}\right) d s\right) \\
= & \int_{\Omega}(\lambda \mathbf{f}+\operatorname{div} \boldsymbol{\sigma}) \cdot \mathbf{u}_{0} d x-\int_{\operatorname{Fr} \Omega} \boldsymbol{\beta}_{B}(\boldsymbol{\sigma}) \cdot \boldsymbol{\gamma}_{B}\left(\mathbf{u}_{0}\right) d s+\lambda \int_{\Gamma_{1}} \mathbf{g} \cdot \boldsymbol{\gamma}_{B}\left(\mathbf{u}_{0}\right) d s \\
= & -\int_{\Gamma_{0}} \boldsymbol{\beta}_{B}(\boldsymbol{\sigma}) \cdot \gamma_{B}\left(\mathbf{u}_{0}\right) d s,
\end{aligned}
$$

where $\operatorname{div} \boldsymbol{\sigma}=-\lambda \mathbf{f}$ in $\Omega$ and $\boldsymbol{\beta}_{B}(\boldsymbol{\sigma})=\lambda \mathbf{g}$ on $\Gamma_{1}$.

Theorem 14 (see [38] and [36]). Suppose $\inf \left(P_{\lambda, j}\right)$ is finite. Moreover, assume that inclusion (6.1) holds. Then $\inf \left(P_{\lambda, j}\right)=\sup \left(P_{\lambda, j}^{*}\right)$ and $\left(P_{\lambda, j}^{*}\right)$ has at least one solution $\sigma_{0} \in W^{n}\left(\Omega\right.$, div), where $\left(P_{\lambda, j}^{*}\right)$ is defined by (6.6), (6.7) and (6.8).

Proof. The function

$$
L^{1}\left(\Omega, \mathbb{E}_{s}^{n}\right) \ni \mathbf{p} \mapsto G_{1, j}(\varepsilon(\mathbf{u})+\mathbf{p}) \in \mathbb{R} \cup\{+\infty\}
$$

is l.s.c. in the topology $\sigma\left(L^{1}\left(\Omega, \mathbb{E}_{s}^{n}\right), L^{\infty}\left(\Omega, \mathbb{E}_{s}^{n}\right)\right)$, where $\mathbf{u} \in L D(\Omega)$. Indeed, by (6.8) and [34, Theorem $3 \mathrm{~A}$ and Proposition $2 \mathrm{M}]$, we get

$$
\begin{aligned}
G_{1, j}^{* *}(\mathbf{p}) & \equiv \sup \left\{\int_{\Omega} \mathbf{p}: \boldsymbol{\sigma} d x-\int_{\Omega} j^{*}(x, \boldsymbol{\sigma}) d x \mid \boldsymbol{\sigma} \in L^{\infty}\left(\Omega, \mathbb{E}_{s}^{n}\right)\right\} \\
& =\int_{\Omega} j(x, \mathbf{p}) d x, \quad \forall \mathbf{p} \in L^{1}\left(\Omega, \mathbb{E}_{s}^{n}\right),
\end{aligned}
$$

since $j^{* *}=j$ (cf. (3.17)). By the Mazur Lemma the function (6.16) is l.s.c. in the norm $\|\cdot\|_{L^{1}}$, because the epigraph of (6.16) is closed in the norm $\mathbb{R} \times L^{1}\left(\Omega, \mathbb{E}_{s}^{n}\right) \ni(z, \mathbf{p}) \mapsto|z|+\|\mathbf{p}\|_{L^{1}\left(\Omega, \mathbb{E}_{s}^{n}\right)}$. By (6.1) we have $j(x, \mathbf{w})=$ $j^{* *}(x, \mathbf{w}) \leq \bar{c}_{n} r_{2}\|\mathbf{w}\|_{\mathbb{E}_{s}^{n}}$ for every $\mathbf{w} \in \mathbb{E}_{s}^{n}$ and for $d x$-a.e. $x \in \Omega$, where the positive constant $\bar{c}_{n}$ depends only on the dimension of $\mathbb{E}_{s}^{n}$. Then $\operatorname{dom} G_{1, j}=$ $L^{1}\left(\Omega, \mathbb{E}_{s}^{n}\right)$. By [17, Chapter 1, Corollary 2.5], the function (6.16) is continuous over the entire space $\left[L^{1}\left(\Omega, \mathbb{E}_{s}^{n}\right),\|\cdot\|_{L^{1}}\right]$. By [17, Chapter 3, Theorem 4.1] the proof is complete.

7. Proofs of basic results. We consider the duality between the displacement formulation and the stress formulation, defined in (4.7) and (4.15). 
Let us recall that the original relaxed problem $\left(R P_{\lambda, j}\right)$ is defined in (4.7). The elastic-plastic potential $G_{j}$ is given by (3.17). Moreover, the dual potential $G_{j}^{*}$ and the bidual potential $G_{j}^{* *}$ are defined in (4.9) and (4.11).

Below we derive an explicit integral representation of $G_{j}^{* *}$ (see Theorem 5). An explicit form of $G_{j}^{*}$ is given in Proposition 4 . We denote by $\varepsilon(B D)(\Omega)$ the set $\left\{\boldsymbol{\mu} \in \mathbb{M}_{b}\left(\Omega, \mathbb{E}_{s}^{n}\right) \mid \exists \mathbf{u} \in B D(\Omega), \boldsymbol{\varepsilon}(\mathbf{u})=\boldsymbol{\mu}\right\}$.

Definition 6. We define

$$
\begin{aligned}
& W_{0}^{n}(\Omega, \operatorname{div}) \equiv\left\{\boldsymbol{\sigma} \in W^{n}(\Omega, \operatorname{div}) \mid \boldsymbol{\beta}_{B}(\boldsymbol{\sigma})=\mathbf{0} \text { on } \operatorname{Fr} \Omega\right\}, \\
& C_{\operatorname{div}}^{c}\left(\Omega, \mathbb{E}_{s}^{n}\right) \equiv C_{c}\left(\Omega, \mathbb{E}_{s}^{n}\right) \cap W_{0}^{n}(\Omega, \operatorname{div}) .
\end{aligned}
$$

We define auxiliary functionals:

$$
G^{*}(\boldsymbol{\sigma}) \equiv \sup \left\{\int_{\Omega} \boldsymbol{\sigma}: \boldsymbol{\mu}-G_{j}(\boldsymbol{\mu}) \mid \boldsymbol{\mu} \in \boldsymbol{\varepsilon}(B D)(\Omega)\right\} \quad \forall \boldsymbol{\sigma} \in W_{0}^{n}(\Omega, \operatorname{div}),
$$

$$
G^{* *}(\varepsilon(\mathbf{u})) \equiv \sup \left\{\int_{\Omega} \boldsymbol{\sigma}: \varepsilon(\mathbf{u})-G^{*}(\boldsymbol{\sigma}) \mid \boldsymbol{\sigma} \in W_{0}^{n}(\Omega, \operatorname{div})\right\} \quad \forall \mathbf{u} \in B D(\Omega) .
$$

Moreover, we define

$$
G_{1}^{\#}(\boldsymbol{\sigma}) \equiv \sup \left\{\int_{\Omega} \boldsymbol{\sigma}: \mathbf{w} d x-G_{j}(\mathbf{w}) \mid \mathbf{w} \in L^{1}\left(\Omega, \mathbb{E}_{s}^{n}\right)\right\} \quad \forall \boldsymbol{\sigma} \in C_{\mathrm{div}}^{c}\left(\Omega, \mathbb{E}_{s}^{n}\right),
$$

$$
G_{1}^{\# \#}(\varepsilon(\mathbf{u})) \equiv \sup \left\{\int_{\Omega} \boldsymbol{\sigma}: \boldsymbol{\varepsilon}(\mathbf{u})-G_{1}^{\#}(\boldsymbol{\sigma}) \mid \boldsymbol{\sigma} \in C_{\mathrm{div}}^{c}\left(\Omega, \mathbb{E}_{s}^{n}\right)\right\} \quad \forall \mathbf{u} \in B D(\Omega),
$$

$$
G^{\#}(\boldsymbol{\sigma}) \equiv \sup \left\{\int_{\Omega} \boldsymbol{\sigma}: \mathbf{w} d x-G_{j}(\mathbf{w}) \mid \mathbf{w} \in \varepsilon(L D(\Omega))\right\} \quad \forall \boldsymbol{\sigma} \in C_{\mathrm{div}}^{c}\left(\Omega, \mathbb{E}_{s}^{n}\right),
$$

$$
G^{\# \#}(\varepsilon(\mathbf{u})) \equiv \sup \left\{\int_{\Omega} \boldsymbol{\sigma}: \boldsymbol{\varepsilon}(\mathbf{u})-G^{\#}(\boldsymbol{\sigma}) \mid \boldsymbol{\sigma} \in C_{\mathrm{div}}^{c}\left(\Omega, \mathbb{E}_{s}^{n}\right)\right\} \quad \forall \mathbf{u} \in B D(\Omega) .
$$

REMARK 2. The definition of spaces placed in duality requires that for every $\boldsymbol{\sigma} \in W_{0}^{n}(\Omega$, div $), \boldsymbol{\sigma} \neq \mathbf{0}$, there exist $\mathbf{M}=\boldsymbol{\varepsilon}(\mathbf{u}) \in \varepsilon(B D)$ such that $\int_{\Omega} \boldsymbol{\sigma}: \mathbf{M}=\int_{\Omega} \boldsymbol{\sigma}: \boldsymbol{\varepsilon}(\mathbf{u}) \neq 0$. But for every $\boldsymbol{\sigma} \in W_{0}^{n}(\Omega$, div $)$ such that $\operatorname{div} \boldsymbol{\sigma}=\mathbf{0}$ in $\Omega$, and for every $\mathbf{M}=\boldsymbol{\varepsilon}(\mathbf{u}) \in \varepsilon(B D)(\Omega)$,

$$
\int_{\Omega} \boldsymbol{\sigma}: \boldsymbol{\varepsilon}(\mathbf{u})=-\int_{\Omega}(\operatorname{div} \boldsymbol{\sigma}) \cdot \mathbf{u} d x+\int_{\operatorname{Fr} \Omega} \boldsymbol{\beta}_{B}(\boldsymbol{\sigma}) \cdot \boldsymbol{\gamma}_{B}^{I}(\mathbf{u}) d s=0
$$

(see (3.9), (5.5) and [19]). Therefore, the definition of the duality should be given between the spaces $\varepsilon(B D)(\Omega)$ and

$$
W_{0}^{n}(\Omega, \operatorname{div}) /\left\{\boldsymbol{\sigma} \in W_{0}^{n}(\Omega, \operatorname{div}) \mid \operatorname{div} \boldsymbol{\sigma}=\mathbf{0} \text { in } \Omega\right\}
$$


or

$$
C_{\text {div }}^{c}\left(\Omega, \mathbb{E}_{s}^{n}\right) /\left\{\boldsymbol{\sigma} \in C_{\operatorname{div}}^{c}\left(\Omega, \mathbb{E}_{s}^{n}\right) \mid \operatorname{div} \boldsymbol{\sigma}=\mathbf{0} \text { in } \Omega\right\} .
$$

For simplicity of proofs, we consider here only the pairs of spaces $(\varepsilon(B D)(\Omega)$, $\left.W_{0}^{n}(\Omega, \operatorname{div})\right)$ and $\left(\varepsilon(B D)(\Omega), C_{\operatorname{div}}^{c}\left(\Omega, \mathbb{E}_{s}^{n}\right)\right)$. Here (7.10) and (7.11) are the sets of equivalence classes for the relation $\operatorname{div}\left(\boldsymbol{\sigma}_{1}-\boldsymbol{\sigma}_{2}\right)=\mathbf{0}$.

We say that the net $\left\{\boldsymbol{\sigma}_{k}\right\}_{k \in \mathbf{K}} \subset C_{\text {div }}^{c}\left(\Omega, \mathbb{E}_{s}^{n}\right)$ converges to $\boldsymbol{\sigma}_{0}$ in the topology $\sigma\left(C_{\text {div }}^{c}\left(\Omega, \mathbb{E}_{s}^{n}\right), L^{1}\left(\Omega, \mathbb{E}_{s}^{n}\right)\right)$ if $\int_{\Omega} \boldsymbol{\sigma}_{k}: \mathbf{w} d x \rightarrow \int_{\Omega} \boldsymbol{\sigma}_{0}: \mathbf{w} d x$ for all $\mathbf{w} \in L^{1}\left(\Omega, \mathbb{E}_{s}^{n}\right)$.

LEMmA 15. Let $f_{1}: C_{\mathrm{div}}^{c}\left(\Omega, \mathbb{E}_{s}^{n}\right) \rightarrow \mathbb{R}$ be a linear functional, continuous in the topology $\sigma\left(C_{\mathrm{div}}^{c}\left(\Omega, \mathbb{E}_{s}^{n}\right), L^{1}\left(\Omega, \mathbb{E}_{s}^{n}\right)\right)$, such that for every $\boldsymbol{\sigma}_{s} \in$ $C_{\operatorname{div}}^{c}\left(\Omega, \mathbb{E}_{s}^{n}\right)$ with $\operatorname{div} \boldsymbol{\sigma}_{s}=\mathbf{0}$ in $\Omega$, we have $f_{1}\left(\boldsymbol{\sigma}_{s}\right)=0$. Then there exists $\widehat{\mathbf{u}}_{1} \in L D(\Omega)$ such that

$$
f_{1}(\boldsymbol{\sigma})=\int_{\Omega} \boldsymbol{\sigma}: \boldsymbol{\varepsilon}\left(\widehat{\mathbf{u}}_{1}\right) d x \quad \forall \boldsymbol{\sigma} \in C_{\operatorname{div}}^{c}\left(\Omega, \mathbb{E}_{s}^{n}\right) .
$$

Proof. Since $f_{1}$ is continuous in the topology $\sigma\left(C_{\text {div }}^{c}\left(\Omega, \mathbb{E}_{s}^{n}\right), L^{1}\left(\Omega, \mathbb{E}_{s}^{n}\right)\right)$, by Theorem V.3.9 of [15] there exists $\mathbf{m} \in L^{1}\left(\Omega, \mathbb{E}_{s}^{n}\right)$ such that $f_{1}(\boldsymbol{\sigma})=$ $\int_{\Omega} \boldsymbol{\sigma}: \mathbf{m} d x$ for all $\boldsymbol{\sigma} \in C_{\text {div }}^{c}\left(\Omega, \mathbb{E}_{s}^{n}\right)$. For every $\boldsymbol{\sigma}_{s} \in C_{\text {div }}^{c}\left(\Omega, \mathbb{E}_{s}^{n}\right)$ such that $\operatorname{div} \boldsymbol{\sigma}_{s}=\mathbf{0}$ in $\Omega$, we have $f_{1}\left(\boldsymbol{\sigma}_{s}\right)=\int_{\Omega} \boldsymbol{\sigma}_{s}: \mathbf{m} d x=0$. Then by [29] and Theorem 1.3 of [36, Chapter 2] there exists $\widehat{\mathbf{u}}_{1} \in L D(\Omega)$ such that (7.12) holds (cf. Proposition 1.1 of [36, Chapter 2]).

Definition 7 (cf. [1]). A subset $H_{0}$ of $\mathcal{L}^{0}\left(\bar{\Omega}, \mathbb{R}^{m}\right)_{\mu_{1}}$ is said to be $P C U$-stable if, for any continuous partition of unity $\left(\alpha_{0}, \ldots, \alpha_{d}\right)$ such that $\alpha_{0}, \ldots, \alpha_{d} \in C^{\infty}(\bar{\Omega}, \mathbb{R})$, and for every $\mathbf{z}_{0}, \ldots, \mathbf{z}_{d} \in H_{0}$, we have $\sum_{i=0}^{d} \alpha_{i} \mathbf{z}_{i}$ $\in H_{0}$.

LEMMA 16. We have

$$
G_{1}^{\# \#}(\varepsilon(\mathbf{u}))=\int_{\Omega} j\left(x, \boldsymbol{\varepsilon}(\mathbf{u})_{a}\right) d x+\int_{\Omega} j_{\infty}\left(x, \frac{d\left(\varepsilon(\mathbf{u})_{s}\right)}{d\left|\boldsymbol{\varepsilon}(\mathbf{u})_{s}\right|}\right) d\left|\varepsilon(\mathbf{u})_{s}\right|
$$

for every $\mathbf{u} \in B D(\Omega)$, where $\varepsilon(\mathbf{u})_{a}$ is the absolutely continuous part and $\boldsymbol{\varepsilon}(\mathbf{u})_{s}$ the singular part of $\boldsymbol{\varepsilon}(\mathbf{u})$ with respect to $d x$.

Proof. By Proposition 4, the proof of [11, Theorem 4'] and formulae (3.10), (3.11), we obtain (7.13) for every $\mathbf{u} \in B D(\Omega)$ (cf. [5. Step 1 of Lemma 4.11]).

Let $Q_{1}: C_{\operatorname{div}}^{c}\left(\Omega, \mathbb{E}_{s}^{n}\right) \rightarrow \mathbb{R} \cup\{+\infty\}$ be defined by

$$
Q_{1}(\boldsymbol{\sigma})=\inf _{\boldsymbol{\sigma}_{s}}\left\{G_{1}^{\#}\left(\boldsymbol{\sigma}+\boldsymbol{\sigma}_{s}\right) \mid \boldsymbol{\sigma}_{s} \in C_{\operatorname{div}}^{c}\left(\Omega, \mathbb{E}_{s}^{n}\right) \text { and } \operatorname{div} \boldsymbol{\sigma}_{s}=\mathbf{0} \text { in } \Omega\right\} .
$$

Proposition 17. For every $\boldsymbol{\sigma} \in C_{\mathrm{div}}^{c}\left(\Omega, \mathbb{E}_{s}^{n}\right)$ we have

$$
G^{\#}(\boldsymbol{\sigma})=\operatorname{cl}_{\sigma\left(C_{\mathrm{div}}^{c}, L^{1}\right)} Q_{1}(\boldsymbol{\sigma}),
$$


where $\operatorname{cl}_{\sigma\left(C_{\mathrm{div}}^{c}, L^{1}\right)} Q_{1}(\boldsymbol{\sigma})$ denotes the largest minorant less than $Q_{1}$ and l.s.c. in the topology $\sigma\left(C_{\mathrm{div}}^{c}\left(\Omega, \mathbb{E}_{s}^{n}\right), L^{1}\left(\Omega, \mathbb{E}_{s}^{n}\right)\right)$ (i.e., $\operatorname{cl}_{\sigma\left(C_{\mathrm{div}}^{c}, L^{1}\right)} Q_{1}$ is the l.s.c. regularization of $Q_{1}$ in $\left.\sigma\left(C_{\mathrm{div}}^{c}, L^{1}\right)\right)$.

Proof (see [7, Proposition 11]). Step 1. By Definition 6, $G^{\#}(\boldsymbol{\sigma}) \leq G_{1}^{\#}(\boldsymbol{\sigma})$ for every $\boldsymbol{\sigma} \in C_{\mathrm{div}}^{c}\left(\Omega, \mathbb{E}_{s}^{n}\right)$. Then $G_{j}(\varepsilon(\mathbf{u})) \geq G^{\# \#}(\varepsilon(\mathbf{u})) \geq G_{1}^{\# \#}(\varepsilon(\mathbf{u}))$ for every $\mathbf{u} \in B D(\Omega)$. Therefore, by (7.13), $G_{j}(\varepsilon(\mathbf{u}))=G^{\# \#}(\varepsilon(\mathbf{u}))=$ $G_{1}^{\# \#}(\varepsilon(\mathbf{u}))$ for every $\mathbf{u} \in L D(\Omega)$.

Step 2. By (3.9) we have

$$
\begin{aligned}
G^{\#}(\boldsymbol{\sigma}) & =\sup \left\{-\int_{\Omega}\left[(\operatorname{div} \boldsymbol{\sigma})+\left(\operatorname{div} \boldsymbol{\sigma}_{s}\right)\right] \cdot \mathbf{u} d x-G_{j}(\boldsymbol{\varepsilon}(\mathbf{u})) \mid \mathbf{u} \in L D(\Omega)\right\} \\
& =G^{\#}\left(\boldsymbol{\sigma}+\boldsymbol{\sigma}_{s}\right)
\end{aligned}
$$

for every $\boldsymbol{\sigma}, \boldsymbol{\sigma}_{s} \in C_{\text {div }}^{c}\left(\Omega, \mathbb{E}_{s}^{n}\right)$ with $\operatorname{div} \boldsymbol{\sigma}_{s}=\mathbf{0}$ in $\Omega$.

Step 3. Suppose there exist $\boldsymbol{\sigma}_{1} \in C_{\mathrm{div}}^{c}\left(\Omega, \mathbb{E}_{s}^{n}\right)$ and a constant $\delta_{0}>0$ such that

$$
G^{\#}\left(\boldsymbol{\sigma}_{1}\right)+\delta_{0}<\mathrm{cl}_{\sigma\left(C_{\mathrm{div}}^{c}, L^{1}\right)} Q_{1}\left(\boldsymbol{\sigma}_{1}\right)
$$

On account of Steps 1 and 2, it suffices to show that this assumption leads to a contradiction.

The linear space $\mathcal{M}_{1} \equiv\left\{\boldsymbol{\sigma}_{s} \in C_{\text {div }}^{c}\left(\Omega, \mathbb{E}_{s}^{n}\right) \mid \operatorname{div} \boldsymbol{\sigma}_{s}=\mathbf{0}\right\}$ is a closed subspace of $\left[C_{\text {div }}^{c}\left(\Omega, \mathbb{E}_{s}^{n}\right), \sigma\left(C_{\operatorname{div}}^{c}\left(\Omega, \mathbb{E}_{s}^{n}\right), L^{1}\left(\Omega, \mathbb{E}_{s}^{n}\right)\right)\right]$. Indeed, by the Green formula (3.9),

$$
\begin{aligned}
\mathcal{M}_{1}= & \bigcap_{\mathbf{u} \in L D(\Omega)}\left\{\boldsymbol{\sigma} \in C_{\mathrm{div}}^{c}\left(\Omega, \mathbb{E}_{s}^{n}\right) \mid\right. \\
& \left.\int_{\Omega} \boldsymbol{\sigma}: \boldsymbol{\varepsilon}(\mathbf{u}) d x=-\int_{\Omega}(\operatorname{div} \boldsymbol{\sigma}) \cdot \mathbf{u} d x+\int_{\operatorname{Fr} \Omega} \boldsymbol{\beta}_{B}(\boldsymbol{\sigma}) \cdot \boldsymbol{\gamma}_{B}(\mathbf{u}) d s=0\right\} .
\end{aligned}
$$

Step 4. Let $\Phi_{1}:\left[C_{\text {div }}^{c}\left(\Omega, \mathbb{E}_{s}^{n}\right), \sigma\left(C_{\text {div }}^{c}, L^{1}\right)\right] \rightarrow C_{\text {div }}^{c}\left(\Omega, \mathbb{E}_{s}^{n}\right) / \mathcal{M}_{1}$ be the linear functional (the canonical homomorphism) such that $\mathcal{M}_{1}=\operatorname{ker} \Phi_{1} \equiv$ $\left\{\boldsymbol{\sigma} \in C_{\mathrm{div}}^{c}\left(\Omega, \mathbb{E}_{s}^{n}\right) \mid \Phi_{1}(\boldsymbol{\sigma})=0\right\}$. Moreover, let $C_{\mathrm{div}}^{c}\left(\Omega, \mathbb{E}_{s}^{n}\right) / \mathcal{M}_{1}$ be endowed with the strongest topology for which $\Phi_{1}$ is continuous. Since $\mathcal{M}_{1}$ is closed in $\sigma\left(C_{\mathrm{div}}^{c}, L^{1}\right)$, the set $C_{\mathrm{div}}^{c}\left(\Omega, \mathbb{E}_{s}^{n}\right) / \mathcal{M}_{1}$ is a Hausdorff topological space (cf. [12. Chapter 1]). Therefore, the point $\left(\Phi_{1}\left(\boldsymbol{\sigma}_{1}\right), G^{\#}\left(\boldsymbol{\sigma}_{1}\right)+\delta_{0}\right)$ is a closed subspace of $\left[C_{\text {div }}^{c}\left(\Omega, \mathbb{E}_{s}^{n}\right) / \mathcal{M}_{1}\right] \times \mathbb{R}$. The epigraph of $\boldsymbol{\sigma} \mapsto \mathrm{cl}_{\sigma\left(C_{\mathrm{div}}^{c}, L^{1}\right)} Q_{1}(\boldsymbol{\sigma})$, defined by

$$
\operatorname{epi~cl}_{\sigma\left(C_{\mathrm{div}}^{c}, L^{1}\right)} Q_{1}=\left\{(\boldsymbol{\sigma}, a) \in C_{\mathrm{div}}^{c}\left(\Omega, \mathbb{E}_{s}^{n}\right) \times \mathbb{R} \mid \mathrm{cl}_{\sigma\left(C_{\mathrm{div}}^{c}, L^{1}\right)} Q_{1}(\boldsymbol{\sigma}) \leq a\right\},
$$

is convex. Then 
$(7.20)$

$\widehat{A}_{1} \equiv\left\{(\widetilde{\boldsymbol{\sigma}}, a) \in\left[C_{\operatorname{div}}^{c}\left(\Omega, \mathbb{E}_{s}^{n}\right) / \mathcal{M}_{1}\right] \times \mathbb{R} \mid \exists \boldsymbol{\sigma}, \operatorname{cl}_{\sigma\left(C_{\mathrm{div}}^{c}, L^{1}\right)} Q_{1}(\boldsymbol{\sigma}) \leq a, \Phi_{1}(\boldsymbol{\sigma})=\tilde{\boldsymbol{\sigma}}\right\}$

is a convex set (cf. [12, Chapter 1]). Moreover $\widehat{A}_{1}$ is a closed subset of $\left[C_{\text {div }}^{c}\left(\Omega, \mathbb{E}_{s}^{n}\right) / \mathcal{M}_{1}\right] \times \mathbb{R}$, since for every $\boldsymbol{\sigma} \in C_{\text {div }}^{c}\left(\Omega, \mathbb{E}_{s}^{n}\right)$ and every $\boldsymbol{\sigma}_{s} \in \mathcal{M}_{1}$, $\operatorname{cl}_{\sigma\left(C_{\mathrm{div}}^{c}, L^{1}\right)} Q_{1}(\boldsymbol{\sigma})=\mathrm{cl}_{\sigma\left(C_{\mathrm{div}}^{c}, L^{1}\right)} Q_{1}\left(\boldsymbol{\sigma}+\boldsymbol{\sigma}_{s}\right)$. By the Hahn-Banach theorem, there exists a closed affine hyperplane $\mathcal{H}$ which strictly separates $\widehat{A}_{1}$ and $\left(\Phi_{1}\left(\boldsymbol{\sigma}_{1}\right), G^{\#}\left(\boldsymbol{\sigma}_{1}\right)+\delta_{0}\right)$. Let

$$
\mathcal{H}=\left\{(\tilde{\boldsymbol{\sigma}}, a) \in\left[C_{\mathrm{div}}^{c}\left(\Omega, \mathbb{E}_{s}^{n}\right) / \mathcal{M}_{1}\right] \times \mathbb{R} \mid f_{2}(\tilde{\boldsymbol{\sigma}})+b a+c_{2}=0\right\}
$$

where $b, c_{2} \in \mathbb{R}$ and $f_{2}: C_{\text {div }}^{c}\left(\Omega, \mathbb{E}_{s}^{n}\right) / \mathcal{M}_{1} \rightarrow \mathbb{R}$ is a continuous linear functional such that for every $(\widetilde{\boldsymbol{\sigma}}, a) \in \widehat{A}_{1}$,

$$
f_{2}\left(\Phi_{1}\left(\boldsymbol{\sigma}_{1}\right)\right)+b\left(G^{\#}\left(\boldsymbol{\sigma}_{1}\right)+\delta_{0}\right)+c_{2}<0<f_{2}(\tilde{\boldsymbol{\sigma}})+b a+c_{2} .
$$

Step 5. Now we consider the case $b=0$. We obtain $G_{1}^{\#}(\boldsymbol{\sigma}) \geq 0$ for every $\boldsymbol{\sigma} \in C_{\mathrm{div}}^{c}\left(\Omega, \mathbb{E}_{s}^{n}\right)$, since $G_{1}^{\#}(\boldsymbol{\sigma})=\int_{\Omega} j^{*}(x, \boldsymbol{\sigma}) d x$ for every $\boldsymbol{\sigma} \in C_{\operatorname{div}}^{c}\left(\Omega, \mathbb{E}_{s}^{n}\right)$ and $j^{*}$ is a non-negative function (cf. Proposition 4). Let

$$
\begin{aligned}
& h \equiv\left|f_{2}\left(\Phi_{1}\left(\boldsymbol{\sigma}_{1}\right)\right)+c_{2}\right|>0, \\
& \widehat{d} \equiv \max \left[1 ; G^{\#}\left(\boldsymbol{\sigma}_{1}\right)+\delta_{0}-\inf _{\boldsymbol{\sigma}}\left\{G_{1}^{\#}(\boldsymbol{\sigma}) \mid \boldsymbol{\sigma} \in C_{\operatorname{div}}^{c}\left(\Omega, \mathbb{E}_{s}^{n}\right)\right\}\right]
\end{aligned}
$$

and $d \equiv h(2 \widehat{d})^{-1}$. Then the functional

$$
\left[C_{\operatorname{div}}^{c}\left(\Omega, \mathbb{E}_{s}^{n}\right) / \mathcal{M}_{1}\right] \times \mathbb{R} \ni(\widetilde{\boldsymbol{\sigma}}, a) \mapsto f_{2}(\widetilde{\boldsymbol{\sigma}})+d\left(a+\widehat{d}-G^{\#}\left(\boldsymbol{\sigma}_{1}\right)-\delta_{0}\right)+c_{2}
$$

strictly separates $\widehat{A}_{1}$ and $\left(\Phi_{1}\left(\boldsymbol{\sigma}_{1}\right), G^{\#}\left(\boldsymbol{\sigma}_{1}\right)+\delta_{0}\right)$.

Step 6. By (7.22) and (7.25) there exist a continuous linear functional $f_{3}: C_{\text {div }}^{c}\left(\Omega, \mathbb{E}_{s}^{n}\right) / \mathcal{M}_{1} \rightarrow \mathbb{R}$ and $c_{3} \in \mathbb{R}$ such that

$$
f_{3}\left(\Phi_{1}\left(\boldsymbol{\sigma}_{1}\right)\right)+c_{3}>G^{\#}\left(\boldsymbol{\sigma}_{1}\right)+\delta_{0} \quad \text { and } \quad f_{3}(\tilde{\boldsymbol{\sigma}})+c_{3}<a
$$

for all $(\widetilde{\boldsymbol{\sigma}}, a) \in \widehat{A}_{1}$. Therefore, the affine functional $\boldsymbol{\sigma} \mapsto f_{4}(\boldsymbol{\sigma})+c_{3}$ defined by $f_{4}(\boldsymbol{\sigma})=f_{3}\left(\Phi_{1}(\boldsymbol{\sigma})\right)$ strictly separates epi $\mathrm{cl}_{\sigma\left(C_{\mathrm{div}}^{c}, L^{1}\right)} Q_{1}$ and

$$
\left\{(\boldsymbol{\sigma}, a) \in C_{\text {div }}^{c}\left(\Omega, \mathbb{E}_{s}^{n}\right) \times \mathbb{R} \mid \boldsymbol{\sigma} \in \mathcal{M}_{1}+\left\{\boldsymbol{\sigma}_{1}\right\}, a=G^{\#}\left(\boldsymbol{\sigma}_{1}\right)+\delta_{0}\right\} .
$$

Moreover, $\mathcal{M}_{1} \subset \operatorname{ker} f_{4}$. Since $\Phi_{1}$ is continuous in the topology $\sigma\left(C_{\mathrm{div}}^{c}, L^{1}\right)$ and $f_{3}$ is continuous on $C_{\operatorname{div}}^{c}\left(\Omega, \mathbb{E}_{s}^{n}\right) / \mathcal{M}_{1}$ it follows that $f_{4}=f_{3} \circ \Phi_{1}$ is continuous in the topology $\sigma\left(C_{\mathrm{div}}^{c}, L^{1}\right)$ over the space $C_{\mathrm{div}}^{c}\left(\Omega, \mathbb{E}_{s}^{n}\right)$.

Step 7. Lemma 15 yields $\widehat{\mathbf{u}}_{1} \in L D(\Omega)$ such that $f_{4}(\boldsymbol{\sigma})=\int_{\Omega} \boldsymbol{\sigma}: \varepsilon\left(\widehat{\mathbf{u}}_{1}\right) d x$ for every $\boldsymbol{\sigma} \in C_{\operatorname{div}}^{c}\left(\Omega, \mathbb{E}_{s}^{n}\right)$, because $\mathcal{M}_{1} \subset \operatorname{ker} f_{4}$.

Step 8. The 1.s.c. regularization of $G_{1}^{\#}$ in the topology $\sigma\left(C_{\operatorname{div}}^{c}\left(\Omega, \mathbb{E}_{s}^{n}\right)\right.$, $\varepsilon(L D(\Omega)))$, denoted by $\mathrm{cl}_{\sigma\left(C_{\mathrm{div}}^{c}, \varepsilon(L D)\right)} G_{1}^{\#}$, is given by 


$$
\begin{array}{r}
\operatorname{cl}_{\sigma\left(C_{\mathrm{div}}^{c}, \boldsymbol{\varepsilon}(L D)\right)} G_{1}^{\#}(\boldsymbol{\sigma})=\sup \left\{\int_{\Omega} \boldsymbol{\sigma}: \boldsymbol{\varepsilon}(\mathbf{u}) d x-G_{1}^{\# \#}(\boldsymbol{\varepsilon}(\mathbf{u})) \mid \mathbf{u} \in L D\right\} \\
=\sup \left\{\int_{\Omega} \boldsymbol{\sigma}: \boldsymbol{\varepsilon}(\mathbf{u}) d x-G_{j}(\boldsymbol{\varepsilon}(\mathbf{u})) \mid \mathbf{u} \in L D\right\}=G^{\#}(\boldsymbol{\sigma})
\end{array}
$$

for every $\boldsymbol{\sigma} \in C_{\mathrm{div}}^{c}\left(\Omega, \mathbb{E}_{s}^{n}\right)$, because $G_{j}(\varepsilon(\mathbf{u}))=G_{1}^{\# \#}(\varepsilon(\mathbf{u}))$ for every $\mathbf{u} \in$ $L D(\Omega)$ (see [17, p. 15], [5, (4.36)] and Step 1). By (7.26), (7.28) and Step 7 of the proof, we obtain a contradiction.

We say that a net $\left\{\boldsymbol{\sigma}_{\tau}\right\}_{\tau \in \mathbf{T}} \subset C_{\text {div }}^{c}\left(\Omega, \mathbb{E}_{s}^{n}\right)$ converges to $\varepsilon_{0}$ in the topology $\sigma\left(C_{\mathrm{div}}^{c}\left(\Omega, \mathbb{E}_{s}^{n}\right), \varepsilon(B D(\Omega))\right)$ if

$$
\int_{\Omega} \boldsymbol{\sigma}_{\tau}: \varepsilon(\mathbf{u}) \rightarrow \int_{\Omega} \boldsymbol{\sigma}_{0}: \varepsilon(\mathbf{u}) \quad \forall \mathbf{u} \in B D(\Omega) .
$$

LEMma 18. For every $\widehat{r}>0$, the topology $\sigma\left(C_{\text {div }}^{c}\left(\Omega, \mathbb{E}_{s}^{n}\right), L^{1}\left(\Omega, \mathbb{E}_{s}^{n}\right)\right)$ is stronger than $\sigma\left(C_{\mathrm{div}}^{c}\left(\Omega, \mathbb{E}_{s}^{n}\right), \varepsilon(B D(\Omega))\right)$ over the set $\left\{\boldsymbol{\sigma} \in C_{\mathrm{div}}^{c}\left(\Omega, \mathbb{E}_{s}^{n}\right) \mid\right.$ $\left.\|\operatorname{div} \boldsymbol{\sigma}\|_{L^{n}\left(\Omega, \mathbb{R}^{n}\right)} \leq \widehat{r}\right\}$.

Proof. Let $\left\{\boldsymbol{\sigma}_{\tau}\right\}_{\tau \in T} \subset\left\{\boldsymbol{\sigma} \in C_{\mathrm{div}}^{c}\left(\Omega, \mathbb{E}_{s}^{n}\right) \mid\|\operatorname{div} \boldsymbol{\sigma}\|_{L^{n}\left(\Omega, \mathbb{R}^{n}\right)} \leq \widehat{r}\right\}$ be a net convergent to $\widehat{\boldsymbol{\sigma}} \in C_{\mathrm{div}}^{c}\left(\Omega, \mathbb{E}_{s}^{n}\right)$ in the topology $\sigma\left(C_{\mathrm{div}}^{c}\left(\Omega, \mathbb{E}_{s}^{n}\right), L^{1}\left(\Omega, \mathbb{E}_{s}^{n}\right)\right)$. Then for every $\mathbf{u} \in L D(\Omega), \int_{\Omega}\left(\boldsymbol{\sigma}_{\tau}-\widehat{\boldsymbol{\sigma}}\right): \boldsymbol{\varepsilon}(\mathbf{u}) d x \rightarrow 0$. By the Green formula (3.9) we have $\int_{\Omega} \operatorname{div}\left(\boldsymbol{\sigma}_{\tau}-\widehat{\boldsymbol{\sigma}}\right) \cdot \mathbf{u} d x \rightarrow 0$ for every $\mathbf{u} \in L D(\Omega)$. The set $L D(\Omega)$ is dense in $\left[L^{n /(n-1)}\left(\Omega, \mathbb{R}^{n}\right),\|\cdot\|_{L^{n /(n-1)}}\right]$, since $C_{c}^{1}\left(\Omega, \mathbb{R}^{n}\right)$ is dense in $L^{n /(n-1)}\left(\Omega, \mathbb{R}^{n}\right)$. Then

$$
\int_{\Omega} \operatorname{div}\left(\boldsymbol{\sigma}_{\tau}-\widehat{\boldsymbol{\sigma}}\right) \cdot \mathbf{w} d x \rightarrow 0 \quad \forall \mathbf{w} \in L^{n /(n-1)}\left(\Omega, \mathbb{R}^{n}\right) .
$$

Indeed, let $\widehat{\mathbf{w}} \in L^{n /(n-1)}\left(\Omega, \mathbb{R}^{n}\right)$ and let $\delta>0$. There exists a sequence $\left\{\widetilde{\mathbf{u}}_{m}\right\}_{m \in \mathbb{N}} \subset L D(\Omega)$ such that $\left\|\widetilde{\mathbf{u}}_{m}-\widehat{\mathbf{w}}\right\|_{L^{n /(n-1)}} \rightarrow 0$. By the Hölder inequality

and

$$
\begin{aligned}
\left|\int_{\Omega} \operatorname{div}\left(\boldsymbol{\sigma}_{\tau}-\widehat{\boldsymbol{\sigma}}\right) \cdot \widehat{\mathbf{w}} d x\right| \leq & \left|\int_{\Omega} \operatorname{div}\left(\boldsymbol{\sigma}_{\tau}-\widehat{\boldsymbol{\sigma}}\right) \cdot\left(\widehat{\mathbf{w}}-\widetilde{\mathbf{u}}_{m}\right) d x\right| \\
& +\left|\int_{\Omega} \operatorname{div}\left(\boldsymbol{\sigma}_{\tau}-\widehat{\boldsymbol{\sigma}}\right) \cdot \widetilde{\mathbf{u}}_{m} d x\right|
\end{aligned}
$$

$$
\begin{aligned}
\left|\int_{\Omega} \operatorname{div}\left(\boldsymbol{\sigma}_{\tau}-\widehat{\boldsymbol{\sigma}}\right) \cdot\left(\widehat{\mathbf{w}}-\widetilde{\mathbf{u}}_{m}\right) d x\right| & \leq\left\|\operatorname{div}\left(\boldsymbol{\sigma}_{\tau}-\widehat{\boldsymbol{\sigma}}\right)\right\|_{L^{n}}\left\|\widehat{\mathbf{w}}-\widetilde{\mathbf{u}}_{m}\right\|_{L^{n /(n-1)}} \\
& \leq 2 \widehat{r}\left\|\widehat{\mathbf{w}}-\widetilde{\mathbf{u}}_{m}\right\|_{L^{n /(n-1)}} \rightarrow 0 \text { as } m \rightarrow \infty .
\end{aligned}
$$

There exists $m_{0} \in \mathbb{N}$ such that for all $\widetilde{m}>m_{0},\left\|\widehat{\mathbf{w}}-\widetilde{\mathbf{u}}_{\widetilde{m}}\right\|_{L^{n /(n-1)}} \leq \delta /(4 \widehat{r})$. Moreover, there exist $\widehat{m}>m_{0}$ and $\tau_{0} \in T$ such that for every $\widetilde{\tau}>\tau_{0}$,

$$
\left|\int_{\Omega} \operatorname{div}\left(\boldsymbol{\sigma}_{\widetilde{\tau}}-\widehat{\boldsymbol{\sigma}}\right) \cdot \widetilde{\mathbf{u}}_{\widehat{m}} d x\right|<\delta / 2
$$

because $\lim _{\tau \in T} \int_{\Omega} \operatorname{div}\left(\boldsymbol{\sigma}_{\tau}-\widehat{\boldsymbol{\sigma}}\right) \cdot \widetilde{\mathbf{u}}_{m} d x=0$ for all $m \in \mathbb{N}$. 
In view of $(7.30), \int_{\Omega} \operatorname{div}\left(\boldsymbol{\sigma}_{\tau}-\widehat{\boldsymbol{\sigma}}\right) \cdot \mathbf{u} d x \rightarrow 0$ for all $\mathbf{u} \in B D(\Omega)$, because $B D(\Omega) \subset L^{n /(n-1)}\left(\Omega, \mathbb{R}^{n}\right)$ (cf. [36, Chapter 2, Theorem 2.2]). By (3.9), the net $\left\{\boldsymbol{\sigma}_{\tau}\right\}_{\tau \in T}$ converges to $\widehat{\boldsymbol{\sigma}}$ in $\sigma\left(C_{\mathrm{div}}^{c}\left(\Omega, \mathbb{E}_{s}^{n}\right), \boldsymbol{\varepsilon}(B D(\Omega))\right)$.

Proposition 19. Let $\widetilde{A}_{t} \equiv\left\{\boldsymbol{\sigma} \in C_{\mathrm{div}}^{c}\left(\Omega, \mathbb{E}_{s}^{n}\right) \mid\|\operatorname{div} \boldsymbol{\sigma}\|_{L^{n}\left(\Omega, \mathbb{R}^{n}\right)} \leq t\right\}$. For every $\widehat{\boldsymbol{\sigma}} \in C_{\operatorname{div}}^{c}\left(\Omega, \mathbb{E}_{s}^{n}\right)$ and every $t>\|\operatorname{div} \widehat{\boldsymbol{\sigma}}\|_{L^{n}}$,

$$
G^{\#}(\widehat{\boldsymbol{\sigma}})=\operatorname{cl}_{\widetilde{A}_{t}} Q_{1}(\widehat{\boldsymbol{\sigma}})
$$

where $\operatorname{cl}_{\widetilde{A}_{t}} Q_{1}(\cdot)$ is the l.s.c. regularization of $\boldsymbol{\sigma} \mapsto Q_{1}(\boldsymbol{\sigma})+I_{\widetilde{A}_{t}}(\boldsymbol{\sigma})$ in the topology $\sigma\left(C_{\mathrm{div}}^{c}\left(\Omega, \mathbb{E}_{s}^{n}\right), L^{1}\left(\Omega, \mathbb{E}_{s}^{n}\right)\right)$, and $I_{\widetilde{A}_{t}}(\cdot)$ is the indicator function of $\widetilde{A}_{t}$.

Proof. Step 1. Suppose there exist $\sigma_{1} \in C_{\text {div }}^{c}\left(\Omega, \mathbb{E}_{s}^{n}\right)$ and constants $t$, $\delta_{0}>0$ such that $t>\left\|\operatorname{div} \boldsymbol{\sigma}_{1}\right\|_{L^{n}}$ and $G^{\#}\left(\boldsymbol{\sigma}_{1}\right)+\delta_{0}<\mathrm{cl}_{\widetilde{A}_{t}} Q_{1}\left(\boldsymbol{\sigma}_{1}\right)$. On account of Steps 1 and 2 of the proof of Proposition 17, it suffices to show that this assumption leads to a contradiction.

For every $\boldsymbol{\varepsilon}(\mathbf{u}) \in \varepsilon(B D(\Omega))$ let

$$
G_{1}^{\# t}(\varepsilon(\mathbf{u})) \equiv \sup \left\{\int_{\Omega} \boldsymbol{\sigma}: \varepsilon(\mathbf{u})-G_{1}^{\#}(\boldsymbol{\sigma}) \mid \boldsymbol{\sigma} \in \widetilde{A}_{t}\right\}
$$

and

$$
G_{1 \| \widetilde{A}_{t}}^{\#}(\boldsymbol{\sigma}) \equiv G_{1}^{\#}(\boldsymbol{\sigma})+I_{\widetilde{A}_{t}}(\boldsymbol{\sigma}) \quad \forall \boldsymbol{\sigma} \in C_{\mathrm{div}}^{c}\left(\Omega, \mathbb{E}_{s}^{n}\right) .
$$

For every $\boldsymbol{\sigma} \in C_{\operatorname{div}}^{c}\left(\Omega, \mathbb{E}_{s}^{n}\right)$ let

$$
\operatorname{cl}_{\sigma\left(C_{\mathrm{div}}^{c}, \varepsilon(L D)\right)} G_{1 \| \widetilde{A}_{t}}^{\#}(\boldsymbol{\sigma})=\sup \left\{\int_{\Omega} \boldsymbol{\sigma}: \boldsymbol{\varepsilon}(\mathbf{u})-G_{1}^{\# t}(\varepsilon(\mathbf{u})) \mid \mathbf{u} \in L D\right\} .
$$

Then for every $\widehat{t}>0$ such that $\left\|\operatorname{div} \sigma_{1}\right\|_{L^{n}}<\widehat{t}$ we have

$$
\operatorname{cl}_{\sigma\left(C_{\mathrm{div}}^{c}, \varepsilon(L D)\right)} G_{1 \| \widetilde{A}_{\bar{t}}^{\#}}^{\#}\left(\boldsymbol{\sigma}_{1}\right)=G^{\#}\left(\boldsymbol{\sigma}_{1}\right)
$$

(cf. (7.28)). Indeed,

$$
\begin{aligned}
\sup \left\{\int_{\Omega} \boldsymbol{\sigma}_{1}: \varepsilon(\mathbf{u})\right. & \left.-G_{1}^{\# t}(\varepsilon(\mathbf{u})) \mid \mathbf{u} \in L D(\Omega)\right\} \\
& =\sup \left\{\int_{\Omega} \boldsymbol{\sigma}_{1}: \varepsilon(\mathbf{u})-G_{1}^{\# \#}(\varepsilon(\mathbf{u})) \mid \mathbf{u} \in L D(\Omega)\right\}
\end{aligned}
$$

if $t>\left\|\operatorname{div} \boldsymbol{\sigma}_{1}\right\|_{L^{n}}$, since $G_{1}^{\# t}$ is the supremum over all affine mappings $\varepsilon(B D(\Omega)) \ni \boldsymbol{\varepsilon}(\mathbf{u}) \mapsto \int_{\Omega} \boldsymbol{\sigma}: \boldsymbol{\varepsilon}(\mathbf{u})+c(\boldsymbol{\sigma})$, such that $c(\boldsymbol{\sigma})=-G_{1}^{\#}(\boldsymbol{\sigma})$ and $\boldsymbol{\sigma} \in \widetilde{A}_{t}$ (cf. (7.6)). By [8, proof of Proposition 13] and Proposition 17 the proof is complete.

Proposition 20. For every $\mathbf{u} \in B D(\Omega)$ we have

$$
G_{1}^{\# \#}(\varepsilon(\mathbf{u}))=G^{\# \#}(\varepsilon(\mathbf{u})) .
$$


Proof. Suppose that there exist $\mathbf{u}_{1} \in B D(\Omega)$ and $\delta_{1}>0$ such that

$$
G^{\# \#}\left(\varepsilon\left(\mathbf{u}_{1}\right)\right)>G_{1}^{\# \#}\left(\varepsilon\left(\mathbf{u}_{1}\right)\right)+4 \delta_{1} .
$$

On account of the proof of Proposition 17, it suffices to show that this assumption leads to a contradiction. There exists $\boldsymbol{\sigma}_{2} \in C_{\mathrm{div}}^{c}\left(\Omega, \mathbb{E}_{s}^{n}\right)$ such that

$$
G^{\# \#}\left(\varepsilon\left(\mathbf{u}_{1}\right)\right)<\left\{\int_{\Omega} \boldsymbol{\sigma}_{2}: \varepsilon\left(\mathbf{u}_{1}\right)-G^{\#}\left(\boldsymbol{\sigma}_{2}\right)\right\}+\delta_{1}
$$

(cf. (7.8)). Therefore, by Lemma 18, Proposition 19, the Green formula (3.9) and [8, (4.49)] there exists $k_{0}>0$ such that

$$
\begin{array}{r}
G^{\# \#}\left(\varepsilon\left(\mathbf{u}_{1}\right)\right)<\left\{-\int_{\Omega}\left(\operatorname{div} \boldsymbol{\sigma}_{2}\right) \cdot \mathbf{u}_{1} d x-\operatorname{cl}_{\widetilde{A}_{k_{0}}} Q_{1}\left(\boldsymbol{\sigma}_{2}\right)\right\}+\delta_{1} \\
\leq \sup _{\boldsymbol{\sigma}} \sup _{\boldsymbol{\sigma}_{s}}\left\{-\int_{\Omega}\left(\operatorname{div}\left(\boldsymbol{\sigma}+\boldsymbol{\sigma}_{s}\right)\right) \cdot \mathbf{u}_{1} d x-G_{1}^{\#}\left(\boldsymbol{\sigma}+\boldsymbol{\sigma}_{s}\right) \mid \boldsymbol{\sigma}, \boldsymbol{\sigma}_{s} \in C_{\operatorname{div}}^{c}\left(\Omega, \mathbb{E}_{s}^{n}\right),\right. \\
\left.\operatorname{div} \boldsymbol{\sigma}_{s}=0 \text { in } \Omega\right\}+\delta_{1}=G_{1}^{\# \#}\left(\varepsilon\left(\mathbf{u}_{1}\right)\right)+\delta_{1} .
\end{array}
$$

By (7.40) we have a contradiction.

Lemma 21 ([5, Lemma 4.12]). The topology $\sigma\left(\varepsilon(B D)(\Omega), W_{0}^{n}(\Omega, \operatorname{div})\right)$ is equivalent to $\sigma\left(\varepsilon(B D)(\Omega), C_{\mathrm{div}}^{c}\left(\Omega, \mathbb{E}_{s}^{n}\right)\right)$ on $\|\cdot\|_{\mathbb{M}_{b}}$-bounded sets from $\varepsilon(B D)(\Omega)$.

Proof. We show that

$$
\left[\operatorname{cl}_{\|\cdot\|_{\mathbb{M}_{b}}} B_{\boldsymbol{\varepsilon}(B D)}(0, r), \sigma\left(\varepsilon(B D)(\Omega), W_{0}^{n}(\Omega, \operatorname{div})\right)\right]
$$

is a compact topological space. Let $\left\{\mathbf{w}_{\delta}\right\}_{\delta \in D} \subset \varepsilon(B D)(\Omega)$ be a $\|\cdot\|_{\mathbb{M}_{b}\left(\Omega, \mathbb{E}_{s}^{n}\right)}$ -bounded net. Then by Proposition 2.3 of [36, Chapter 2] there exists a $\|\cdot\|_{B D(\Omega)}$-bounded net $\left\{\mathbf{u}_{\delta}\right\}_{\delta \in D}$ such that for every $\delta \in D, \varepsilon\left(\mathbf{u}_{\delta}\right)=\mathbf{w}_{\delta}$. Since $B D(\Omega) \subset L^{n /(n-1)}\left(\Omega, \mathbb{R}^{n}\right)$ (cf. [36, Theorem 2.2, Chapter 2]), we deduce that there exist a finer net $\left\{\mathbf{u}_{\delta_{\alpha}}\right\}_{\alpha \in A} \subset\left\{\mathbf{u}_{\delta}\right\}_{\delta \in D}$ and a function $\mathbf{u}_{0} \in$ $L^{n /(n-1)}\left(\Omega, \mathbb{R}^{n}\right)$ such that

$$
\int_{\Omega} \boldsymbol{\sigma}: \boldsymbol{\varepsilon}\left(\mathbf{u}_{\delta_{\alpha}}\right)=-\int_{\Omega}(\operatorname{div} \boldsymbol{\sigma}) \mathbf{u}_{\delta_{\alpha}} d x \rightarrow-\int_{\Omega}(\operatorname{div} \boldsymbol{\sigma}) \mathbf{u}_{0} d x
$$

for every $\boldsymbol{\sigma} \in W_{0}^{n}(\Omega$, div) (see (3.9)). Moreover, there exist a finer net $\left\{\mathbf{u}_{\delta_{\alpha_{\beta}}}\right\}_{\beta \in B}$ and $\boldsymbol{\mu}_{0} \in \mathbb{M}_{b}\left(\Omega, \mathbb{E}_{s}^{n}\right)$ such that $\boldsymbol{\varepsilon}\left(\mathbf{u}_{\delta_{\alpha_{\beta}}}\right) \rightarrow \boldsymbol{\mu}_{0}$ in the topology $\sigma\left(\mathbb{M}_{b}\left(\Omega, \mathbb{E}_{s}^{n}\right), C_{0}\left(\Omega, \mathbb{E}_{s}^{n}\right)\right)$. The symmetric distributional derivative $\boldsymbol{\varepsilon}\left(\mathbf{u}_{0}\right)$ of $\mathbf{u}_{0}$ is equal to $\boldsymbol{\mu}_{0}$. Thus $\mathbf{u}_{0} \in B D(\Omega)$. Therefore, the topological space (7.43) 
is compact, since $\left\|\varepsilon\left(\mathbf{u}_{\delta}\right)\right\|_{\mathbb{M}_{b}\left(\Omega, \mathbb{E}_{s}^{n}\right)} \leq r$ for every $\delta \in D$ and

$$
\begin{aligned}
\left\|\varepsilon\left(\mathbf{u}_{0}\right)\right\|_{\mathbb{M}_{b}\left(\Omega, \mathbb{E}_{s}^{n}\right)}=\sup _{\varphi}\left\{\int_{\Omega} \varphi: \varepsilon\left(\mathbf{u}_{0}\right) \mid \varphi \in C_{\operatorname{div}}^{c}\left(\Omega, \mathbb{E}_{s}^{n}\right),\right. \\
\\
\left.\left|\varphi_{i j}(x)\right| \leq 1 \forall i, j \in\{1, \ldots, n\}, \forall x \in \Omega\right\}
\end{aligned}
$$

(cf. (3.3) and [36, Chapter 2, (2.1)]). The Hausdorff topology $\sigma(\varepsilon(B D)(\Omega)$, $\left.C_{\text {div }}^{c}\left(\Omega, \mathbb{E}_{s}^{n}\right)\right)$ is weaker than $\sigma\left(\varepsilon(B D)(\Omega), W_{0}^{n}(\Omega, \operatorname{div})\right)$. Therefore, these topologies are equivalent on bounded sets in $\varepsilon(B D)$ (see [18, Corollary 3.1.14]).

Note that the set $\mathrm{cl}_{\|\cdot\|_{\mathbb{M}_{b}}} B_{\boldsymbol{\varepsilon}(B D)(\Omega)}(0, r)$ with the topology $\sigma(\varepsilon(B D)$, $W^{n}(\Omega$, div) ) is not compact (cf. [5, Lemma 4.12]). Indeed, if $\Omega=(0,1)$ and $\left\{w_{m}\right\}_{m \in \mathbb{N}} \subset \varepsilon(B D)(0,1)$ such that $w_{m}$ is the Dirac delta at the point $x_{m}=1 / m$, then $\left\{w_{m}\right\}_{m \in \mathbb{N}}$ converges to $\delta_{0}$, where $\delta_{0}$ is the Dirac delta at 0 . But $\delta_{0} \notin \varepsilon(B D)(0,1)$, because $0 \notin(0,1)$. The sequence $\left\{w_{m}\right\}_{m \in \mathbb{N}}$ converges to $0 \in \varepsilon(B D)(0,1)$ in the topology $\sigma\left(\varepsilon(B D)(0,1), W_{0}^{1}((0,1)\right.$, div $\left.\left.)\right)\right)$. Moreover, $\left\langle w_{m}, 1_{(0,1)}\right\rangle_{Y \times W_{0}((0,1) \text {,div })}=\int_{0}^{1} 1 w_{m}=1$ for every $m \in \mathbb{N}$, where $1_{(0,1)}$ is the constant function equal to 1 on the interval $(0,1)$.

Theorem 22 (cf. Theorem 4.14 of [5]). $G^{\# \#}(\varepsilon(\mathbf{u}))=G_{1}^{\# \#}(\varepsilon(\mathbf{u}))=$ $G^{* *}(\varepsilon(\mathbf{u}))$ for every $\mathbf{u} \in B D(\Omega)$.

Proof. The function $G^{* *}$ is the l.s.c. regularization of $G_{j \mid \varepsilon(B D)}$ in the topology $\sigma\left(\varepsilon(B D), W_{0}^{n}(\Omega, \operatorname{div})\right)$. Moreover, if $\left\|\mathbf{z}_{m}\right\|_{L^{1}} \rightarrow+\infty$, then $G_{j}\left(\mathbf{z}_{m}\right)$ $\rightarrow+\infty$ (cf. (5.6)). Therefore, by (7.3), (7.4), (7.7), (7.8) and Lemma 21, we find $G^{\# \#}=G^{* *}$.

Proposition 23. We have, for every $\mathbf{u} \in B D(\Omega)$ (cf. (4.9)),

$$
\begin{aligned}
\widetilde{G}(\varepsilon(\mathbf{u})) & \equiv \sup _{\boldsymbol{\sigma}}\left\{\int_{\Omega} \boldsymbol{\sigma}: \boldsymbol{\varepsilon}(\mathbf{u})-G_{j}^{*}(\boldsymbol{\sigma}) \mid \boldsymbol{\sigma} \in W_{0}^{n}(\Omega, \operatorname{div})\right\} \\
& =\int_{\Omega} j\left(x, \boldsymbol{\varepsilon}(\mathbf{u})_{a}\right) d x+\int_{\Omega} j_{\infty}\left(x, \frac{d\left(\varepsilon(\mathbf{u})_{s}\right)}{d\left|\varepsilon(\mathbf{u})_{s}\right|}\right) d\left|\varepsilon(\mathbf{u})_{s}\right| .
\end{aligned}
$$

Proof. Step 1. We have $G_{j}^{*}(\boldsymbol{\sigma}) \geq G^{*}(\boldsymbol{\sigma})$ for every $\boldsymbol{\sigma} \in W_{0}^{n}(\Omega, \operatorname{div})$, since in the definition of $G_{j}^{*}$ we take the supremum over a larger domain (see (4.2), (4.9) and (7.3)). Then $\widetilde{G} \leq G^{* *}$ (i.e., $\widetilde{G}(\varepsilon(\mathbf{u})) \leq G^{* *}(\varepsilon(\mathbf{u}))$ for every $\mathbf{u} \in B D(\Omega)$ ); see also Lemma 16 and Theorem 22 .

Step 2. Define $\widehat{G}_{j}: \varepsilon(B D)(\Omega) \rightarrow \mathbb{R} \cup\{+\infty\}$ by

$$
\widehat{G}_{j}(\varepsilon(\mathbf{u})) \equiv \sup \left\{\int_{\Omega} \boldsymbol{\sigma}: \boldsymbol{\varepsilon}(\mathbf{u})-\int_{\Omega} j^{*}(x, \boldsymbol{\sigma}) d x \mid \boldsymbol{\sigma} \in C_{\operatorname{div}}^{c}\left(\Omega, \mathbb{E}_{s}^{n}\right)\right\} .
$$

By Proposition 4 , we have $\widehat{G}_{j} \leq \widetilde{G}$, since $C_{\mathrm{div}}^{c} \subset W_{0}^{n}\left(\Omega\right.$, div). Since $C_{\mathrm{div}}^{c}$ is 
PCU-stable (cf. Definition 7), by [11, Theorems 1 and 4] we get

$$
\begin{aligned}
\widehat{G}_{j}(\varepsilon(\mathbf{u})) \equiv & \sup \left\{\int_{\Omega} \boldsymbol{\sigma}: \boldsymbol{\varepsilon}(\mathbf{u})_{a} d x-\int_{\Omega} j^{*}(x, \boldsymbol{\sigma}) d x+\int_{\Omega} \boldsymbol{\sigma}: \boldsymbol{\varepsilon}(\mathbf{u})_{s} \mid\right. \\
& \left.\boldsymbol{\sigma} \in C_{\mathrm{div}}^{c}, \boldsymbol{\sigma}(x) \in \mathcal{K}(x), \forall x \in \Omega\right\} \\
= & \sup \left\{\int_{\Omega}\left[\boldsymbol{\sigma}: \boldsymbol{\varepsilon}(\mathbf{u})_{a}-j^{*}(x, \boldsymbol{\sigma})\right] d x\right. \\
& \left.+\int_{\Omega}\left[\boldsymbol{\sigma}: \frac{d\left(\boldsymbol{\varepsilon}(\mathbf{u})_{s}\right)}{d\left|\varepsilon(\mathbf{u})_{s}\right|}-I_{\mathcal{K}(x)}(\boldsymbol{\sigma})\right] d\left|\boldsymbol{\varepsilon}(\mathbf{u})_{s}\right| \mid \boldsymbol{\sigma} \in C_{\mathrm{div}}^{c}\left(\Omega, \mathbb{E}_{s}^{n}\right)\right\} \\
= & \int_{\Omega} j\left(x, \boldsymbol{\varepsilon}(\mathbf{u})_{a}\right) d x+\int_{\Omega} j_{\infty}\left(x, \frac{d\left(\varepsilon(\mathbf{u})_{s}\right)}{d\left|\boldsymbol{\varepsilon}(\mathbf{u})_{s}\right|}\right) d\left|\varepsilon(\mathbf{u})_{s}\right|
\end{aligned}
$$

(see (3.10), (3.11), (7.13) and Theorem 22).

Proof of Theorem 5. Step 1. The distribution $\boldsymbol{\sigma}: \boldsymbol{\varepsilon}(\mathbf{u})$ is an absolutely continuous measure with respect to $|\varepsilon(\mathbf{u})|$ and

$$
\left|\int_{\Omega} \varphi \boldsymbol{\sigma}: \varepsilon(\mathbf{u})\right| \leq\|\boldsymbol{\sigma}\|_{L^{\infty}\left(\Omega, \mathbb{E}_{s}^{n}\right)} \int_{\Omega}|\varphi| d|\varepsilon(\mathbf{u})|
$$

for every $\boldsymbol{\sigma} \in W^{n}\left(\Omega, \operatorname{div}\right.$ ), $\mathbf{u} \in B D(\Omega)$ and $\varphi \in C_{c}(\Omega)$ (cf. [36, Chapter 2, Lemma 7.3]). Then, by the Radon-Nikodym Theorem (for given $\mathbf{u} \in B D(\Omega)$ and $\left.\boldsymbol{\sigma} \in W^{n}(\Omega, \operatorname{div})\right)$, there is a Borel-measurable function $\tau^{\mathbf{u}, \boldsymbol{\sigma}}: \Omega \rightarrow \mathbb{R}$ such that for every Borel subset $\Omega_{b}$ of $\Omega$,

$$
\int_{\Omega_{b}} \boldsymbol{\sigma}: \varepsilon(\mathbf{u})=\int_{\Omega_{b}} \tau^{\mathbf{u}, \boldsymbol{\sigma}}|\varepsilon(\mathbf{u})|
$$

since $|\varepsilon(\mathbf{u})|$ and $\boldsymbol{\sigma}: \varepsilon(\mathbf{u})$ are Borel measures.

Step 2. Let $\mathbf{u} \in B D(\Omega)$. Moreover, let $\Omega_{a}$ and $\Omega_{s}$ be Borel subsets of $\Omega$ such that $\Omega=\Omega_{a} \cup \Omega_{s}, \Omega_{a} \cap \Omega_{s}=\emptyset$ and $\left|\varepsilon(\mathbf{u})_{a}\right|\left(\Omega_{s}\right)=0=\left|\varepsilon(\mathbf{u})_{s}\right|\left(\Omega_{a}\right)$, where $\varepsilon(\mathbf{u})_{a}$ is the absolutely continuous part and $\varepsilon(\mathbf{u})_{s}$ the singular part of $\varepsilon(\mathbf{u})$ with respect to $d x$. Similarly to [2, Theorem 3.1] we obtain $\tau^{\mathbf{u}, \boldsymbol{\sigma}}(x)=$ $\boldsymbol{\sigma}(x): \frac{d\left(\boldsymbol{\varepsilon}(\mathbf{u})_{a}\right)}{d\left|\boldsymbol{\varepsilon}(\mathbf{u})_{a}\right|}(x)$ for $d x$-a.e. $x \in \Omega_{a}$, where $\frac{d\left(\boldsymbol{\varepsilon}(\mathbf{u})_{a}\right)}{d\left|\boldsymbol{\varepsilon}(\mathbf{u})_{a}\right|}(\widetilde{x})=\mathbf{0}$ for $d x$-a.e. $\widetilde{x} \in\left\{x \in \Omega \mid \varepsilon(\mathbf{u})_{a}(x)=\mathbf{0}\right\}$.

Step 3. Let us recall that $\operatorname{sgn}(z)=1$ if $z>0, \operatorname{sgn}(z)=0$ if $z=0$ and $\operatorname{sgn}(z)=-1$ if $z<0$. For given $\mathbf{u} \in B D(\Omega)$ and $\boldsymbol{\sigma} \in W^{n}(\Omega$, div $)-$ $C_{\text {div }}^{c}\left(\Omega, \mathbb{E}_{s}^{n}\right)$ we define a Borel-measurable function $\boldsymbol{\sigma}_{\mathbf{u}}: \Omega \rightarrow \mathbb{E}_{s}^{n}$ by

$$
\boldsymbol{\sigma}_{\mathbf{u}}(x)= \begin{cases}\boldsymbol{\sigma}(x) & \text { for } d x \text {-a.e. } x \in \Omega_{a}, \\ \tau^{\mathbf{u}, \boldsymbol{\sigma}}(x)\left[\operatorname{sgn}\left(\frac{d\left(\varepsilon(\mathbf{u})_{s}\right)_{i j}}{d\left|\varepsilon(\mathbf{u})_{s}\right|}\right)\right](x) & \text { for }\left|\varepsilon(\mathbf{u})_{s}\right| \text {-a.e. } x \in \Omega_{s},\end{cases}
$$


where $\left[\operatorname{sgn}\left(\frac{d\left(\boldsymbol{\varepsilon}(\mathbf{u})_{s}\right)_{i j}}{d\left|\boldsymbol{\varepsilon}(\mathbf{u})_{s}\right|}\right)\right]$ is the matrix whose $i j$-entry is $\operatorname{sgn}\left(\frac{d\left(\boldsymbol{\varepsilon}(\mathbf{u})_{s}\right)_{i j}}{d\left|\boldsymbol{\varepsilon}(\mathbf{u})_{s}\right|}\right)$. If $\boldsymbol{\sigma} \in$ $C_{\text {div }}^{c}\left(\Omega, \mathbb{E}_{s}^{n}\right)$ then $\boldsymbol{\sigma}_{\mathbf{u}}=\boldsymbol{\sigma}$. Thus we obtain $\tau^{\mathbf{u}, \boldsymbol{\sigma}}(x)=\boldsymbol{\sigma}_{\mathbf{u}}(x): \frac{d(\boldsymbol{\varepsilon}(\mathbf{u}))}{d|\boldsymbol{\varepsilon}(\mathbf{u})|}(x)$ for $\left(d x+\left|\varepsilon(\mathbf{u})_{s}\right|\right)$-a.e. $x \in \Omega$. Here, by (7.51), the following function is defined: $B D(\Omega) \times W^{n}(\Omega, \operatorname{div}) \ni(\mathbf{u}, \boldsymbol{\sigma}) \mapsto \boldsymbol{\sigma}_{\mathbf{u}} \in \mathcal{L}^{0}\left(\Omega, \mathbb{E}_{s}^{n}\right)_{d x+\left|\varepsilon(\mathbf{u})_{s}\right|} \cdot$

Step 4. If $\boldsymbol{\sigma}^{1}, \boldsymbol{\sigma}^{2} \in W^{n}(\Omega, \operatorname{div})$ and $\varphi^{1}, \varphi^{2} \in C^{\infty}(\bar{\Omega}, \mathbb{R})$, then for every Borel subset $\Omega_{b}$ of $\Omega$,

$$
\begin{array}{r}
\int_{\Omega_{b}}\left(\varphi^{1} \tau^{\mathbf{u}, \boldsymbol{\sigma}^{1}}+\varphi^{2} \tau^{\mathbf{u}, \boldsymbol{\sigma}^{2}}\right)|\varepsilon(\mathbf{u})|=\int_{\Omega_{b}} \varphi^{1}\left(\boldsymbol{\sigma}^{1}: \boldsymbol{\varepsilon}(\mathbf{u})\right)+\int_{\Omega_{b}} \varphi^{2}\left(\boldsymbol{\sigma}^{2}: \boldsymbol{\varepsilon}(\mathbf{u})\right) \\
=\int_{\Omega_{b}}\left(\varphi^{1} \boldsymbol{\sigma}^{1}+\varphi^{2} \boldsymbol{\sigma}^{2}\right): \boldsymbol{\varepsilon}(\mathbf{u})=\int_{\Omega_{b}} \tau^{\mathbf{u},\left(\varphi^{1} \boldsymbol{\sigma}^{1}+\varphi^{2} \boldsymbol{\sigma}^{2}\right)}|\boldsymbol{\varepsilon}(\mathbf{u})| .
\end{array}
$$

Step 5. For the given $\mathbf{u} \in B D(\Omega)$ and for

$$
H_{1} \equiv\left\{\boldsymbol{\sigma} \in W^{n}(\Omega, \operatorname{div}) \mid \boldsymbol{\sigma}(x) \in \mathcal{K}(x) \text { for } d x \text {-a.e. } x \in \Omega\right\}
$$

there exists a smallest closed-valued measurable multifunction $\Gamma^{\mathbf{u}}$ such that $\boldsymbol{\sigma}_{\mathbf{u}}(x) \in \Gamma^{\mathbf{u}}(x)$ for every $\boldsymbol{\sigma} \in H_{1}$ and for $\left(d x+\left|\boldsymbol{\varepsilon}(\mathbf{u})_{s}\right|\right)$-a.e. $x \in \Omega$ (see [11] and [40]). Let $H_{0}$ be given by (7.53) with $W^{n}(\Omega$, div) replaced by $W_{0}^{n}(\Omega, \operatorname{div})$. Then for given $\mathbf{u} \in B D(\Omega)$ there exists a smallest closed-valued measurable multifunction $\Gamma_{0}^{\mathbf{u}}$ such that $\boldsymbol{\sigma}_{\mathbf{u}}(x) \in \Gamma_{0}^{\mathbf{u}}(x)$ for every $\boldsymbol{\sigma} \in H_{0}$ and for $\left(d x+\left|\varepsilon(\mathbf{u})_{s}\right|\right)$-a.e. $x \in \Omega$. Let us define

$$
\begin{aligned}
\widetilde{H}_{1}^{\mathbf{u}} \equiv\left\{\boldsymbol{\sigma}_{\mathbf{u}} \in \mathcal{L}^{0}\left(\Omega, \mathbb{R}^{m}\right)_{d x+\left|\boldsymbol{\varepsilon}(\mathbf{u})_{s}\right|} \mid \boldsymbol{\sigma} \in W^{n}(\Omega, \operatorname{div})\right. \\
\left.\boldsymbol{\sigma}_{\mathbf{u}}(x)=\boldsymbol{\sigma}(x) \in \mathcal{K}(x) \text { for } d x \text {-a.e. } x \in \Omega\right\}
\end{aligned}
$$

and let $\widetilde{H}_{0}^{\mathbf{u}}$ be given by $(7.54)$ with $\left.W^{n}(\Omega, \operatorname{div})\right)$ replaced by $W_{0}^{n}(\Omega, \operatorname{div})$. By Step $4, \widetilde{H}_{1}^{\mathbf{u}}$ and $\widetilde{H}_{0}^{\mathbf{u}}$ are PCU-stable sets, since $\mathcal{K}(x)$ is convex for every $x \in \bar{\Omega}$. Because of [11, Theorem 1] we obtain

$$
\begin{aligned}
& \int_{\Omega_{s}} \operatorname{ess}_{\mathbf{w} \in \Gamma^{\mathbf{u}}(x)}\left[\mathbf{w}: \frac{d\left(\varepsilon(\mathbf{u})_{s}\right)}{d\left|\varepsilon(\mathbf{u})_{s}\right|}\right] d\left|\varepsilon(\mathbf{u})_{s}\right| \\
& +\int_{\Omega_{a}} \operatorname{essiup}_{\mathbf{w} \in \Gamma^{\mathbf{u}}(x)}\left[\mathbf{w}: \boldsymbol{\varepsilon}(\mathbf{u})_{a}-j^{*}(x, \mathbf{w})\right] d x \\
& \geq \int_{\Omega_{s}} \operatorname{ess}_{\mathbf{w} \in \Gamma_{0}^{\mathbf{u}}(x)}\left[\mathbf{w}: \frac{d\left(\varepsilon(\mathbf{u})_{s}\right)}{d\left|\varepsilon(\mathbf{u})_{s}\right|}\right] d\left|\varepsilon(\mathbf{u})_{s}\right|+\int_{\Omega_{a}} \operatorname{ess}_{\mathbf{w} \in \Gamma_{0}^{\mathbf{u}}(x)}\left[\mathbf{w}: \varepsilon(\mathbf{u})_{a}-j^{*}(x, \mathbf{w})\right] d x
\end{aligned}
$$$$
=\sup \left\{\int_{\Omega} \boldsymbol{\sigma}: \boldsymbol{\varepsilon}(\mathbf{u})-\int_{\Omega} j^{*}(x, \boldsymbol{\sigma}) d x \mid \boldsymbol{\sigma} \in W_{0}^{n}(\Omega, \operatorname{div})\right\} .
$$ 
Let the Borel-measurable function $h: \Omega \rightarrow \mathbb{R} \cup\{+\infty\}$ be given by

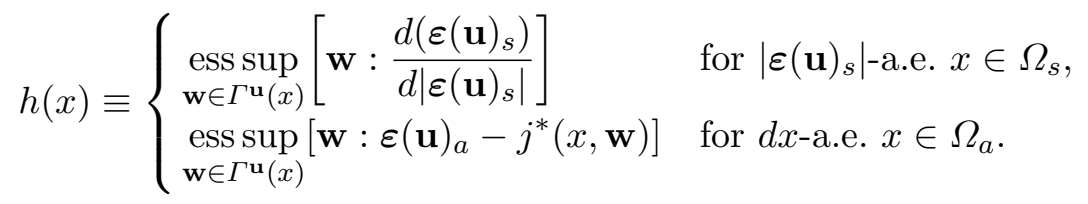

Similarly, let $h_{0}: \Omega \rightarrow \mathbb{R} \cup\{+\infty\}$ be defined by (7.56), where $\Gamma^{\mathbf{u}}$ is replaced by $\Gamma_{0}^{\mathbf{u}}$. For $\left(d x+\left|\varepsilon(\mathbf{u})_{s}\right|\right)$-a.e. $x \in \Omega, h(x) \geq h_{0}(x)$, since $\Gamma_{0}^{\mathbf{u}}(x) \subset \Gamma^{\mathbf{u}}(x)$.

Step 6. There exists an increasing sequence $\left\{\Omega^{k}\right\}_{k \in \mathbb{N}}$ of closed sets such that $\bigcup_{k=1}^{\infty} \Omega^{k}=\Omega, \Omega^{k_{1}} \subset \Omega^{k_{2}}$ if $k_{1}<k_{2}$,

$$
\forall k \in \mathbb{N}, \quad\left|\varepsilon(\mathbf{u})_{s}\right|\left(\Omega-\Omega^{k}\right)<\frac{1}{2 k} \quad \text { and } \quad d x\left(\Omega-\Omega^{k}\right)<\frac{1}{2 k},
$$

since $|\varepsilon(\mathbf{u})|$ and $d x$ are regular measures. Since the space $\mathbb{R}^{n}$ is normal, for every $\Omega^{k}=\overline{\Omega^{k}} \subset \Omega$ there exists a continuous function $\widetilde{t}^{k}: \mathbb{R}^{n} \rightarrow[0,1]$ such that $\widetilde{t}_{\mid \Omega^{k}}^{k}=1$ and $\widetilde{t}_{\mid \mathbb{R}^{n}-\Omega}^{k}=0$ (cf. Theorem 1.5.10 of [18]). Therefore, we can assume that there exists an increasing sequence $\left\{\Omega^{k}\right\}_{k \in \mathbb{N}}$ of closed sets such that $\bigcup_{k=1}^{\infty} \Omega^{k}=\Omega, \overline{\Omega^{k_{1}}}=\Omega^{k_{1}} \subset \operatorname{int} \Omega^{k_{2}}$ if $k_{1}<k_{2}$ and condition (7.57) holds.

Step 7. By Urysohn's lemma [18, Theorem 1.5.10] for every $k \in \mathbb{N}$ there exists a continuous function $t_{k}: \mathbb{R}^{n} \rightarrow[0,1]$ such that $t_{k \mid \Omega^{k+1}}=1$ and $t_{k \mid \mathbb{R}^{n}-\text { int } \Omega^{k+2}}=0$, because $\overline{\Omega^{k+1}}=\Omega^{k+1} \subset \operatorname{int} \Omega^{k+2}$. There exists $\varrho>0$ such that

(7.58) $\operatorname{dist}\left(\operatorname{Fr} \Omega^{k}, \operatorname{Fr} \Omega^{k+1}\right) \equiv \inf \left\{\|x-y\|_{\mathbb{R}^{n}} \mid x \in \operatorname{Fr} \Omega^{k}, y \in \operatorname{Fr} \Omega^{k+1}\right\}>2 \varrho$ and $\operatorname{dist}\left(\operatorname{Fr} \Omega^{k+2}, \operatorname{Fr} \Omega^{k+3}\right)>2 \varrho$, since $\Omega$ is bounded and $\overline{\Omega^{k_{1}}} \subset \operatorname{int} \Omega^{k_{2}}$ for every $k_{1}<k_{2}$. Therefore, there exists a non-negative function $J_{\varrho} \in$ $C^{\infty}(\mathbb{R},[0, \infty))$ such that $\int_{\mathbb{R}^{n}} J_{\varrho}(x) d x=1$ and $J_{\varrho}(x)=0$ if $\|x\|_{\mathbb{R}^{n}} \geq \varrho$. The function $\widehat{t}_{k}: \mathbb{R}^{n} \rightarrow[0,1]$, defined to be the convolution $J_{\varrho} * t_{k}(y)=$ $\int_{\mathbb{R}^{n}} J_{\varrho}(y-x) t_{k}(x) d x$, is smooth, $\widehat{t}_{k \mid \Omega^{k}}=1$ and $\widehat{t}_{k \mid \mathbb{R}^{n}-\operatorname{int} \Omega^{k+3}}=0$. By (3.7), for every $k \in \mathbb{N}$ and every $\boldsymbol{\sigma} \in W^{n}(\Omega$, div) such that $\boldsymbol{\sigma}(x) \in \mathcal{K}(x)$ for $d x$-a.e. $x \in \Omega$, we have $\widehat{t}_{k} \boldsymbol{\sigma} \in W_{0}^{n}(\Omega, \operatorname{div})$ and $\widehat{t}_{k}(x) \boldsymbol{\sigma}(x) \in \mathcal{K}(x)$ for $d x$-a.e. $x \in \Omega$, because $0 \in \mathcal{K}(x)$ for all $x \in \bar{\Omega}$ (see (3.11), (5.1)). Then $\Gamma_{0}^{\mathbf{u}}(x)=\Gamma^{\mathbf{u}}(x)$ for $\left(d x+\left|\varepsilon(\mathbf{u})_{s}\right|\right)$-a.e. $x \in \Omega^{k}$ and for every $k \in \mathbb{N}$. By $(7.56), h(x)=h_{0}(x)$ for $\left(d x+\left|\varepsilon(\mathbf{u})_{s}\right|\right)$-a.e. $x \in \Omega^{k}$ and for every $k \in \mathbb{N}$. Then $h(x)=h_{0}(x)$ for $\left(d x+\left|\varepsilon(\mathbf{u})_{s}\right|\right)$-a.e. $x \in \Omega$. From (7.55), (7.56) and Propositions 4 and 23 we obtain (4.12).

The proof of Proposition 6 is based on the following result: 
Lemma 24. For every $\boldsymbol{\sigma} \in W^{n}(\Omega$, div) we have

$$
\begin{aligned}
& \sup \left\{-\int_{\Gamma_{0}} \boldsymbol{\beta}_{B}(\boldsymbol{\sigma}) \cdot \gamma_{B}(\mathbf{u}) d s-\int_{\Gamma_{0}} j_{\infty}\left(x,\left(\mathbf{u}^{0}-\boldsymbol{\gamma}_{B}(\mathbf{u})\right) \otimes_{s} \boldsymbol{\nu}\right) d s \mid\right. \\
& \mathbf{u} \in B D(\Omega)\}= \begin{cases}-\int_{\Gamma_{0}} \boldsymbol{\beta}_{B}(\boldsymbol{\sigma}) \cdot \mathbf{u}^{0} d s & \text { if } \boldsymbol{\beta}_{B}(\boldsymbol{\sigma})(x) \in \mathcal{K}(x) \cdot \boldsymbol{\nu}(x) \\
+\infty & \text { for ds-a.e. } x \in \Gamma_{0},\end{cases}
\end{aligned}
$$

Proof. Let $\mathbf{t} \in \mathbb{R}^{n}$; then for $d s$-a.e. $x \in \Gamma_{0}$,

$$
\begin{aligned}
j_{\infty}\left(x,\left(\mathbf{t} \otimes_{s} \boldsymbol{\nu}(x)\right)\right) & =\sup \left\{\widehat{\boldsymbol{\sigma}}:\left(\mathbf{t} \otimes_{s} \boldsymbol{\nu}(x)\right) \mid \widehat{\boldsymbol{\sigma}} \in \mathcal{K}(x) \subset \mathbb{E}_{s}^{n}\right\} \\
& =\sup \{(\widehat{\boldsymbol{\sigma}} \cdot \boldsymbol{\nu}(x)) \cdot \mathbf{t} \mid \widehat{\boldsymbol{\sigma}} \cdot \boldsymbol{\nu}(x) \in \mathcal{K} \cdot \boldsymbol{\nu}(x)\},
\end{aligned}
$$

because for every $\widehat{\boldsymbol{\sigma}}$ (such that $\widehat{\boldsymbol{\sigma}} \cdot \boldsymbol{\nu}(x) \in \mathcal{K}(x) \cdot \boldsymbol{\nu}(x)$ ) there exists $\overline{\boldsymbol{\sigma}} \in$ $\mathcal{K}(x)$ such that $\overline{\boldsymbol{\sigma}} \cdot \boldsymbol{\nu}(x)=\widehat{\boldsymbol{\sigma}} \cdot \boldsymbol{\nu}(x)$. The function $\Gamma_{0} \times \mathbb{R}^{n} \ni(x, \mathbf{t}) \mapsto$ $\bar{j}_{\infty}(x, \mathbf{t})=j_{\infty}\left(x,\left(\mathbf{t} \otimes_{s} \boldsymbol{\nu}(x)\right)\right)$ is a non-negative, convex, normal integrand (i.e., conditions (i) and (ii) from Definition 1 hold $d s$-a.e. for $\bar{j}_{\infty}: \Gamma_{0} \times \mathbb{R}^{n}$ $\rightarrow \mathbb{R}$, cf. [17, p. 232]). Indeed, by Theorem 26 from Appendix A, there exists a multifunction $\mathcal{K}^{\prime}: \Gamma_{0} \rightarrow 2^{\mathbb{E}_{s}^{n}}$ such that $\mathcal{K}(x)=\mathcal{K}^{\prime}(x)$ for $d s$-a.e. $x \in \Gamma_{0}$ and $\left\{(x, \mathbf{y}) \in \Gamma_{0} \times \mathbb{E}_{s}^{n} \mid \mathbf{y} \in \mathcal{K}^{\prime}(x)\right\}$ is a Borel subset of $\Gamma_{0} \times \mathbb{E}_{s}^{n}$. Therefore, $\Gamma_{0} \times \mathbb{E}_{s}^{n} \ni(x, \mathbf{y}) \mapsto I_{\mathcal{K}^{\prime}(x)}(\mathbf{y}) \in \mathbb{R} \cup\{+\infty\}$ is a Borel function. For every $x \in \Gamma_{0}, \mathbb{E}_{s}^{n} \ni \mathbf{y} \mapsto I_{\mathcal{K}(x)}(\mathbf{y})$ is l.s.c., since $\mathcal{K}(x)$ is a closed subset in $\mathbb{E}_{s}^{n}$. By [17, Proposition 1.2, p. 237], $j_{\infty}: \Gamma_{0} \times \mathbb{E}_{s}^{n} \rightarrow \mathbb{R} \cup\{+\infty\}$ is a normal integrand. Therefore, $\bar{j}_{\infty}: \Gamma_{0} \times \mathbb{R}^{n} \rightarrow \mathbb{R} \cup\{+\infty\}$ is a convex, normal integrand, because $\Omega$ is $C^{1}$ and $\Gamma_{0} \times \mathbb{R}^{n} \ni(x, \mathbf{t}) \mapsto\left(x, \mathbf{t} \otimes_{s} \boldsymbol{\nu}(x)\right) \in \Gamma_{0} \times \mathbb{E}_{s}^{n}$ is continuous. By [34, Theorem $3 \mathrm{~A}]$, since $\gamma_{B}$ is a surjection onto $L^{1}\left(\operatorname{Fr} \Omega, \mathbb{R}^{n}\right)$, we find

$$
\begin{aligned}
\sup \left\{\int_{\Gamma_{0}} \boldsymbol{\beta}_{B}(\boldsymbol{\sigma}) \cdot\left(\mathbf{u}^{0}-\boldsymbol{\gamma}_{B}(\mathbf{u})\right) d s-\int_{\Gamma_{0}} \bar{j}_{\infty}\left(x,\left(\mathbf{u}^{0}-\boldsymbol{\gamma}_{B}(\mathbf{u})\right)\right) d s \mid\right. \\
\left.\boldsymbol{\gamma}_{B}(\mathbf{u}) \in L^{1}\left(\operatorname{Fr} \Omega, \mathbb{R}^{n}\right)\right\}-\int_{\Gamma_{0}} \boldsymbol{\beta}_{B}(\boldsymbol{\sigma}) \cdot \mathbf{u}^{0} d s=\text { RHS of }(7.59) .
\end{aligned}
$$

Proof of Proposition 6. As the space $\left\{(\mathbf{w}, \mathbf{z}) \in L^{1}\left(\Omega, \mathbb{R}^{n}\right) \times L^{1}\left(\operatorname{Fr} \Omega, \mathbb{R}^{n}\right) \mid\right.$ $\left.\exists \mathbf{u} \in B D(\Omega), \mathbf{u}=\mathbf{w}, \gamma_{B}(\mathbf{u})=\mathbf{z}\right\}$ is PCU-stable, by Theorem 1 of [11] and by Lemma 24 we get

$$
\begin{aligned}
& \left(F_{\lambda, R}\right)^{*}\left(-\boldsymbol{\varepsilon}^{*}(\boldsymbol{\sigma})\right) \\
& =\sup \left\{\int_{\Omega}(\operatorname{div} \boldsymbol{\sigma}+\lambda \mathbf{f}) \cdot \mathbf{u} d x+\int_{\Gamma_{1}}\left(\lambda \mathbf{g}-\boldsymbol{\beta}_{B}(\boldsymbol{\sigma})\right) \cdot \boldsymbol{\gamma}_{B}(\mathbf{u}) d s\right. \\
& \quad+\int_{\Gamma_{0}} \boldsymbol{\beta}_{B}(\boldsymbol{\sigma}) \cdot\left(\mathbf{u}^{0}-\boldsymbol{\gamma}_{B}(\mathbf{u})\right) d s-\int_{\Gamma_{0}} j_{\infty}\left(x,\left(\mathbf{u}^{0}-\boldsymbol{\gamma}_{B}(\mathbf{u})\right) \otimes_{s} \boldsymbol{\nu}\right) d s \mid \\
& \mathbf{u} \in B D(\Omega)\}-\int_{\Gamma_{0}} \boldsymbol{\beta}_{B}(\boldsymbol{\sigma}) \cdot \mathbf{u}^{0} d s=\text { RHS of }(4.13) .
\end{aligned}
$$


Proof of Proposition 7. Similarly to the proof of Proposition 6, we find

$$
\begin{aligned}
& \left(F_{\lambda, R}\right)^{*}\left(\mathbf{u}^{*}, \boldsymbol{\varphi}^{*}\right) \\
& \quad= \begin{cases}\int_{\Gamma_{0}} \boldsymbol{\varphi}^{*} \cdot \mathbf{u}^{0} d s & \text { if } \mathbf{u}^{*}=\lambda \mathbf{f}, \\
& \boldsymbol{\varphi}^{*}(x)=-\lambda \mathbf{g}(x) \text { for } d s \text {-a.e. } x \in \Gamma_{1} \\
& \text { and }-\boldsymbol{\varphi}^{*}(x) \in \mathcal{K}(x) \cdot \boldsymbol{\nu}(x) \text { for } d s \text {-a.e. } x \in \Gamma_{0}, \\
+\infty & \text { otherwise. }\end{cases}
\end{aligned}
$$

Then by (7.60) we have

$$
\begin{aligned}
&\left(F_{\lambda, R}\right)^{* *}(\mathbf{u})= \sup \left\{\left\langle\mathbf{u},\left(\mathbf{u}^{*}, \boldsymbol{\varphi}^{*}\right)\right\rangle_{B D \times V^{*}}-\int_{\Gamma_{0}} \boldsymbol{\varphi}^{*} \cdot \mathbf{u}^{0} d s \mid\right. \\
&\left(\mathbf{u}^{*}, \boldsymbol{\varphi}^{*}\right) \in V^{*}, \mathbf{u}^{*}=\lambda \mathbf{f}, \boldsymbol{\varphi}^{*}=-\lambda \mathbf{g} \text { on } \Gamma_{1}, \\
&\left.-\boldsymbol{\varphi}^{*}(x) \in \mathcal{K}(x) \cdot \boldsymbol{\nu}(x) \text { for } d s \text {-a.e. } x \in \Gamma_{0}\right\}=F_{\lambda, R}(\mathbf{u})
\end{aligned}
$$

for every $\mathbf{u} \in B D(\Omega)$.

Proposition 25. If $\boldsymbol{\sigma}_{1} \in W^{n}\left(\Omega\right.$, div) and $\boldsymbol{\sigma}_{1}(x) \in \mathcal{K}(x)$ for $d x$-a.e. $x \in \Omega$, then $\boldsymbol{\beta}_{B}\left(\boldsymbol{\sigma}_{1}\right)(x) \in \mathcal{K}(x) \cdot \boldsymbol{\nu}(x)$ for ds-a.e. $x \in \operatorname{Fr} \Omega$.

Proof. Step 1. Let $\mathcal{K}_{1}: \bar{\Omega}_{1} \rightarrow 2^{\mathbb{E}_{s}^{n}}$ be given by $\mathcal{K}_{1}(x)=\mathcal{K}(x)$ for every $x \in \bar{\Omega}$ and $\mathcal{K}_{1}(x)=\mathbb{E}_{s}^{n}$ for every $x \in \bar{\Omega}_{1}-\bar{\Omega}$ (cf. Assumptions 1 and 2 ). Let $j_{1}^{*}: \Omega_{1} \times \mathbb{E}_{s}^{n} \rightarrow \mathbb{R} \cup\{+\infty\}$ be the normal integrand defined by

$$
j_{1}^{*}\left(x, \mathbf{w}^{*}\right)= \begin{cases}\left\|\mathbf{w}^{*}\right\|_{\mathbb{E}_{s}^{n}}^{2} & \text { if } \mathbf{w}^{*} \in \mathcal{K}_{1}(x), \\ +\infty & \text { if } \mathbf{w}^{*} \notin \mathcal{K}_{1}(x),\end{cases}
$$

for every $x \in \Omega_{1}$ and every $\mathbf{w}^{*} \in \mathbb{E}_{s}^{n}$ (cf. Theorem 26 from Appendix A and Remark 1). The original potential $j_{1}: \Omega_{1} \times \mathbb{E}_{s}^{n} \rightarrow \mathbb{R} \cup\{+\infty\}$ is defined by

$$
j_{1}(x, \mathbf{w})=\sup \left\{\mathbf{w}: \mathbf{w}^{*}-j_{1}^{*}\left(x, \mathbf{w}^{*}\right) \mid \mathbf{w}^{*} \in \mathbb{E}_{s}^{n}\right\}
$$

for every $\mathbf{w} \in \mathbb{E}_{s}^{n}$ and $d x$-a.e. $x \in \Omega_{1}$. The functional $G_{j_{1}}: \mathbb{M}_{b}\left(\Omega_{1}, \mathbb{E}_{s}^{n}\right) \rightarrow$ $\mathbb{R} \cup\{+\infty\}$ is given by (3.17) with $j$ replaced by $j_{1}$. Due to Proposition 4 ,

$$
\begin{aligned}
G_{j_{1}}^{*}(\boldsymbol{\sigma}) & \equiv \sup _{\mathbf{w}}\left\{\int_{\Omega_{1}}\left[\boldsymbol{\sigma}: \mathbf{w}-j_{1}(x, \mathbf{w})\right] d x \mid \mathbf{w} \in L^{1}\left(\Omega_{1}, \mathbb{E}_{s}^{n}\right)\right\} \\
& =\int_{\Omega_{1}} j_{1}^{*}(x, \boldsymbol{\sigma}) d x
\end{aligned}
$$

for every $\boldsymbol{\sigma} \in W^{n}\left(\Omega_{1}\right.$, div $)$. Let the bidual functional $G_{j_{1}}^{* \#}: \varepsilon\left(B D\left(\Omega_{1}\right)\right) \rightarrow$ $\mathbb{R} \cup\{+\infty\}$ be defined by

$$
G_{j_{1}}^{* \#}(\varepsilon(\mathbf{u})) \equiv \sup _{\boldsymbol{\sigma}}\left\{\int_{\Omega_{1}} \boldsymbol{\sigma}: \varepsilon(\mathbf{u})-G_{j_{1}}^{*}(\boldsymbol{\sigma}) \mid \boldsymbol{\sigma} \in C_{\mathrm{div}}^{c}\left(\Omega_{1}, \mathbb{E}_{s}^{n}\right)\right\}
$$


for every $\mathbf{u} \in B D\left(\Omega_{1}\right)$. Because of Lemma 16,

$$
G_{j_{1}}^{* \#}(\varepsilon(\mathbf{u}))=\int_{\Omega_{1}} j_{1}\left(x, \boldsymbol{\varepsilon}(\mathbf{u})_{a}\right) d x+\int_{\Omega_{1}}\left(j_{1}\right)_{\infty}\left(x, \frac{d\left(\varepsilon(\mathbf{u})_{s}\right)}{d\left|\varepsilon(\mathbf{u})_{s}\right|}\right) d\left|\varepsilon(\mathbf{u})_{s}\right|
$$

for every $\mathbf{u} \in B D\left(\Omega_{1}\right)$. Moreover, define $\widetilde{G}_{j_{1}}: \varepsilon\left(B D\left(\Omega_{1}\right)\right) \rightarrow \mathbb{R} \cup\{+\infty\}$ by (7.70)

$$
\widetilde{G}_{j_{1}}(\varepsilon(\mathbf{u})) \equiv \sup _{\boldsymbol{\sigma}}\left\{\int_{\Omega_{1}} \boldsymbol{\sigma}: \varepsilon(\mathbf{u})-G_{j_{1}}^{*}(\boldsymbol{\sigma}) \mid \boldsymbol{\sigma} \in W_{0}^{n}\left(\Omega_{1}, \operatorname{div}\right)\right\}
$$

for every $\mathbf{u} \in B D\left(\Omega_{1}\right)$. By Proposition $23, \widetilde{G}_{j_{1}}(\varepsilon(\mathbf{u}))=G_{j_{1}}^{* \#}(\varepsilon(\mathbf{u}))$ for every $\mathbf{u} \in B D\left(\Omega_{1}\right)$.

Step 2. Similarly to the proof of Theorem 5 , for every $\mathbf{u} \in B D\left(\Omega_{1}\right)$ and $\boldsymbol{\sigma} \in W_{0}^{n}\left(\Omega_{1}\right.$, div), there exists a Borel-measurable function $\tau^{\mathbf{u}, \boldsymbol{\sigma}}: \Omega_{1} \rightarrow \mathbb{R}$ such that for every Borel subset $\Omega_{b}$ of $\Omega_{1}, \int_{\Omega_{b}} \boldsymbol{\sigma}: \varepsilon(\mathbf{u})=\int_{\Omega_{b}} \tau, \mathbf{u}, \boldsymbol{\sigma}|\varepsilon(\mathbf{u})|$. Moreover, let $\boldsymbol{\sigma}_{\mathbf{u}}$ be given by (7.51). For the given $\mathbf{u} \in B D\left(\Omega_{1}\right)$ and for

$$
H_{0}^{\Omega_{1}} \equiv\left\{\boldsymbol{\sigma} \in W_{0}^{n}\left(\Omega_{1}, \operatorname{div}\right) \mid \boldsymbol{\sigma}(x) \in \mathcal{K}_{1}(x) \text { for } d x \text {-a.e. } x \in \Omega_{1}\right\},
$$

there exists a smallest closed-valued measurable multifunction $\Gamma_{\Omega_{1}}^{\mathbf{u}}$ such that $\boldsymbol{\sigma}_{\mathbf{u}}(x) \in \Gamma_{\Omega_{1}}^{\mathbf{u}}(x)$ for every $\boldsymbol{\sigma} \in H_{0}^{\Omega_{1}}$ and for $\left(d x+\left|\varepsilon(\mathbf{u})_{s}\right|\right)$-a.e. $x \in \Omega_{1}$. By $(5.5)$,

$$
\int_{\operatorname{Fr} \Omega} \tau^{\mathbf{u}, \boldsymbol{\sigma}}|\varepsilon(\mathbf{u})|=\int_{\operatorname{Fr} \Omega} \boldsymbol{\sigma}: \boldsymbol{\varepsilon}(\mathbf{u})=\int_{\operatorname{Fr} \Omega} \boldsymbol{\beta}_{B}\left(\boldsymbol{\sigma}_{\mid \Omega}\right) \cdot\left(\boldsymbol{\gamma}_{B}^{O}(\mathbf{u})-\gamma_{B}^{I}(\mathbf{u})\right) d s
$$

for every $\mathbf{u} \in B D\left(\Omega_{1}\right)$ and $\boldsymbol{\sigma} \in W_{0}^{n}\left(\Omega_{1}\right.$, div). Moreover, by Step 3 of the proof of Theorem 5 we get $\int_{\mathrm{Fr} \Omega} \boldsymbol{\sigma}_{\mathbf{u}}: \boldsymbol{\varepsilon}(\mathbf{u})=\int_{\mathrm{Fr} \Omega} \tau^{\mathbf{u}, \boldsymbol{\sigma}}|\varepsilon(\mathbf{u})|$ for every $\mathbf{u} \in$ $B D\left(\Omega_{1}\right)$ and $\boldsymbol{\sigma} \in W_{0}^{n}\left(\Omega_{1}\right.$, div $)$.

Step 3. Let there exist $\widehat{\boldsymbol{\sigma}} \in W^{n}(\Omega$, div) such that $\widehat{\boldsymbol{\sigma}}(x) \in \mathcal{K}(x)$ for $d x$-a.e. $x \in \Omega$ and $\boldsymbol{\beta}_{B}(\widehat{\boldsymbol{\sigma}})(x) \notin \mathcal{K}(x) \cdot \boldsymbol{\nu}(x)$ for $x \in \omega \subset \operatorname{Fr} \Omega$ with $d s(\omega)>0$. There exists $\widehat{\mathbf{u}} \in B D\left(\Omega_{1}\right)$ such that

$$
\int_{\operatorname{Fr} \Omega} \boldsymbol{\beta}_{B}(\widehat{\boldsymbol{\sigma}}) \cdot\left(\boldsymbol{\gamma}_{B}^{O}(\widehat{\mathbf{u}})-\gamma_{B}^{I}(\widehat{\mathbf{u}})\right) d s>
$$

$\sup _{\boldsymbol{\sigma}}\left\{\int_{\mathrm{Fr} \Omega} \boldsymbol{\beta}_{B}\left(\left.\boldsymbol{\sigma}\right|_{\Omega}\right) \cdot\left(\boldsymbol{\gamma}_{B}^{O}(\widehat{\mathbf{u}})-\boldsymbol{\gamma}_{B}^{I}(\widehat{\mathbf{u}})\right) d s \mid \boldsymbol{\sigma} \in C_{\mathrm{div}}^{c}, \boldsymbol{\sigma}(x) \in \mathcal{K}_{1}(x) \forall x \in \Omega_{1}\right\}$,

since $\gamma_{B}$ is a surjection on $L^{1}\left(\operatorname{Fr} \Omega, \mathbb{R}^{n}\right)$. Then, by (7.72), we obtain

$$
\int_{\operatorname{Fr} \Omega} \widehat{\boldsymbol{\sigma}}_{\mathbf{u}}:\left[\left(\boldsymbol{\gamma}_{B}^{O}(\widehat{\mathbf{u}})-\boldsymbol{\gamma}_{B}^{I}(\widehat{\mathbf{u}})\right) \otimes_{s} \boldsymbol{\nu}\right] d s>
$$

$$
\sup _{\boldsymbol{\sigma}}\left\{\int_{\operatorname{Fr} \Omega} \boldsymbol{\sigma}:\left[\left(\boldsymbol{\gamma}_{B}^{O}(\widehat{\mathbf{u}})-\boldsymbol{\gamma}_{B}^{I}(\widehat{\mathbf{u}})\right) \otimes_{s} \boldsymbol{\nu}\right] d s \mid \boldsymbol{\sigma} \in C_{\mathrm{div}}^{c}, \boldsymbol{\sigma}(x) \in \mathcal{K}_{1}(x) \forall x \in \Omega_{1}\right\} .
$$

Hence $\Gamma_{\Omega_{1}}^{\mathbf{u}}(x) \cdot \boldsymbol{\nu}(x) \nsubseteq \mathcal{K}_{1}(x) \cdot \boldsymbol{\nu}(x)$ for $d s$-a.e. $x \in \omega \subset \operatorname{Fr} \Omega$ and $\mathcal{K}_{1}(\widetilde{x}) \cdot \boldsymbol{\nu}(\widetilde{x}) \subset$ $\Gamma_{\Omega_{1}}^{\mathrm{u}}(\widetilde{x}) \cdot \boldsymbol{\nu}(\widetilde{x})$ for $d s$-a.e. $\widetilde{x} \in \operatorname{Fr} \Omega$, since $C_{\operatorname{div}}^{c}\left(\Omega_{1}, \mathbb{E}_{s}^{n}\right) \subset W_{0}^{n}(\Omega, \operatorname{div})$ and by 
[5. Lemma 2.13] (see also (7.60) and Remark 1). Because of (7.55) we get $\widetilde{G}_{j_{1}}(\varepsilon(\widehat{\mathbf{u}}))>G_{j_{1}}^{* \#}(\varepsilon(\widehat{\mathbf{u}}))$. Thus we have a contradiction (see Step 1).

Now Proposition 4, Proposition 6 and Section 2 imply Theorem 8. Moreover, from Theorem 5, Proposition 7, formula (4.8) and Section 2, Theorem 9 follows.

Because of Lemma 13 and Proposition 25, the problems $\left(R P_{\lambda, j}^{*}\right)$ and $\left(P_{\lambda, j}^{*}\right)$ are equivalent.

8. Appendix A. In this section we prove an auxiliary result.

Theorem 26 (cf. [34, Theorem $1 \mathrm{~F}]$ ). Let $\Omega_{2}$ be a bounded, open set of class $C^{1}$ in $\mathbb{R}^{n}$ and let the multifunction $\mathcal{K}_{0}: \bar{\Omega}_{2} \rightarrow 2^{\mathbb{E}_{s}^{n}}$ satisfy the following condition:

$$
\begin{array}{r}
\mathcal{K}_{0}(y)=\left\{\mathbf{z}(y) \in \mathbb{E}_{s}^{n} \mid \mathbf{z} \in C^{t}\left(\bar{\Omega}_{2}, \mathbb{E}_{s}^{n}\right), \mathbf{z}_{\mid \text {int } \Omega_{2}} \in W^{n}\left(\Omega_{2}, \operatorname{div}\right),\right. \\
\left.\mathbf{z}(x) \in \mathcal{K}_{0}(x) \text { for } d x \text {-a.e. } x \in \Omega_{2}\right\}
\end{array}
$$

for all $y \in \bar{\Omega}_{2}$, where $t$ is a fixed integer $(t \geq 0)$. Moreover, let $\mathcal{K}_{0}(x)$ be a convex and closed subset in $\mathbb{E}_{s}^{n}$ for all $x \in \bar{\Omega}_{2}$. Then:

(i) for every $\delta>0$, there is a closed set $T_{\delta} \subset \bar{\Omega}_{2}$ with $d x\left(\Omega_{2}-T_{\delta}\right)+$ $d s\left(\operatorname{Fr} \Omega_{2}-T_{\delta}\right)<\delta$ such that the set $\left\{(x, \mathbf{w}) \in \bar{\Omega}_{2} \times \mathbb{E}_{s}^{n} \mid x \in T_{\delta}\right.$, $\left.\mathbf{w} \in \mathcal{K}_{0}(x)\right\}$ is closed;

(ii) there is a closed-valued multifunction $\mathcal{K}_{0}^{\prime}: \bar{\Omega}_{2} \rightarrow 2^{\mathbb{E}_{s}^{n}}$ such that the graph of $\mathcal{K}_{0}^{\prime} \equiv\left\{(x, \mathbf{w}) \in \bar{\Omega}_{2} \times \mathbb{E}_{s}^{n} \mid \mathbf{w} \in \mathcal{K}_{0}^{\prime}(x)\right\}$ is a Borel set in $\bar{\Omega}_{2} \times \mathbb{E}_{s}^{n}, \mathcal{K}_{0}^{\prime}(x)=\mathcal{K}_{0}(x)$ for $d x$-a.e. $x \in \Omega_{2}$ and $\mathcal{K}_{0}^{\prime}(x)=\mathcal{K}_{0}(x)$ for ds-a.e. $x \in \operatorname{Fr} \Omega_{2}$.

Proof. Step 1. Since $\Omega_{2}$ is bounded we have $d x\left(\Omega_{2}\right)<\infty$. The boundary $\operatorname{Fr} \Omega_{2}$ is a closed, bounded subset of $\mathbb{R}^{n}$ since $\Omega_{2}$ is of class $C^{1}$. Hence $d s\left(\operatorname{Fr} \Omega_{2}\right)<\infty$.

Step 2. Let $\left\{C_{m}\right\}_{m \in \mathbb{N}}$ be an enumeration of all countably many closed subsets of $\mathbb{E}_{s}^{n}$, complementary to open balls with rational centers and radii. For each $x \in \bar{\Omega}_{2}, \mathcal{K}_{0}(x)$ is the intersection of all the sets $C_{m}$ containing $x$. Let $S_{m}=\left\{x \in \bar{\Omega}_{2} \mid \mathcal{K}_{0}(x) \cap\left(\mathbb{E}_{s}^{n}-C_{m}\right) \neq \emptyset\right\}$ and $S_{m}^{\prime}=\bar{\Omega}_{2}-S_{m}=\left\{x \in \bar{\Omega}_{2} \mid\right.$ $\left.\mathcal{K}_{0}(x) \subset C_{m}\right\}$. By [40] (see also [6, Theorem 7.1]) there exists a sequence $\left\{\mathbf{z}_{i}\right\}_{i \in \mathbb{N}} \subset C^{t}\left(\bar{\Omega}_{2}, \mathbb{E}_{s}^{n}\right)$ such that $\mathbf{z}_{i \mid \operatorname{int} \Omega_{2}} \in W^{n}\left(\Omega_{2}\right.$, div) for every $i \in \mathbb{N}$ and

$$
\mathcal{K}_{0}(y)=\operatorname{cl}_{\mathbb{E}_{s}^{n}}\left\{\mathbf{z}(y) \in \mathbb{E}_{s}^{n} \mid \mathbf{z} \in\left\{\mathbf{z}_{i}\right\}_{i \in \mathbb{N}}\right\}
$$

for $d x$-a.e. $y \in \Omega_{2}$ and for $d s$-a.e. $y \in \operatorname{Fr} \Omega_{2}\left(\right.$ or $\mathcal{K}_{0}(y)=\emptyset$ for every $\left.y \in \Omega_{2}\right)$. Then by [34, Theorem 1B] the multifunction $\mathcal{K}_{0}$ is measurable. Therefore, 
$S_{m}$ and $S_{m}^{\prime}$ are measurable sets for every $m \in \mathbb{N}$ (cf. [34]) and

$$
\text { graph of } \mathcal{K}_{0}=\bigcap_{m=1}^{\infty}\left[\left(S_{m} \times \mathbb{E}_{s}^{n}\right) \cup\left(S_{m}^{\prime} \times C_{m}\right)\right] \text {. }
$$

Fix $\delta>0$. For each $m$, there exist compact sets $\omega_{m} \subset S_{m}$ and $\omega_{m}^{\prime} \subset S_{m}^{\prime}$ such that

$$
\begin{array}{r}
d x\left(\Omega_{2}-\left(\omega_{m} \cup \omega_{m}^{\prime}\right)\right) \leq \delta\left(2^{-(m+1)}\right), \\
d s\left(\operatorname{Fr} \Omega_{2}-\left(\omega_{m} \cup \omega_{m}^{\prime}\right)\right) \leq \delta\left(2^{-(m+1)}\right),
\end{array}
$$

since $d x$ over $\Omega_{2}$ and $d s$ over $\operatorname{Fr} \Omega_{2}$ are regular measures. Let

$$
T_{\delta}=\bigcap_{m=1}^{\infty}\left(\omega_{m} \cup \omega_{m}^{\prime}\right)
$$

Then $T_{\delta}$ is a compact set with $d x\left(\Omega_{2}-T_{\delta}\right)+d s\left(\operatorname{Fr} \Omega_{2}-T_{\delta}\right)<\delta$, and we have

$$
\left\{(x, \mathbf{w}) \in \bar{\Omega}_{2} \times \mathbb{E}_{s}^{n} \mid x \in T_{\delta}, \mathbf{w} \in \mathcal{K}_{0}(x)\right\}=\bigcap_{m=1}^{\infty}\left[\left(\omega_{m} \times \mathbb{E}_{s}^{n}\right) \cup\left(\omega_{m}^{\prime} \times C_{m}\right)\right] .
$$

The latter set is closed, so (i) is established.

Step 3. For $\delta=m^{-1}, m=1,2, \ldots$, let $T$ be the union of the corresponding sequence of sets $T_{\delta}$. Then $T$ is measurable with $d x\left(\Omega_{2}-T\right)+d s\left(\operatorname{Fr} \Omega_{2}-T\right)$ $=0$, and the set $\left\{(x, \mathbf{w}) \in \bar{\Omega}_{2} \times \mathbb{E}_{s}^{n} \mid x \in T, \mathbf{w} \in \mathcal{K}_{0}(x)\right\}$ is a union of closed sets. Thus (ii) is satisfied with $\mathcal{K}_{0}^{\prime}$ equal to the restriction of $\mathcal{K}_{0}$ to $T$.

\section{References}

[1] E. Acerbi, I. Fonseca and N. Fusco, Regularity results for equilibria in a variational model for fracture, Proc. Roy. Soc. Edinburgh Sect. A 127 (1997), 889-902.

[2] G. Anzellotti, On the extremal stress and displacement in Hencky plasticity, Duke Math. J. 51 (1984), 133-147.

[3] G. Anzellotti and M. Giaquinta, Convex functionals and partial regularity, Arch. Ration. Mech. Anal. 102 (1988), 243-272.

[4] G. Bellettini, A. Coscia and G. Dal Maso, Compactness and lower semicontinuity properties in $S B D(\Omega)$, Math. Z. 228 (1998), 337-351.

[5] J. L. Bojarski, The relaxation of Signorini problems in Hencky plasticity. I: threedimensional solid, Nonlinear Anal. 29 (1997), 1091-1116.

[6] —, The relaxation of Signorini problems in Hencky plasticity. II: plates, ibid. 29 (1997), 1117-1143.

[7] —, Regularity of solutions in plasticity. I: Continuum, Appl. Math. (Warsaw) 30 (2003), 337-364.

[8] —, General method of regularization. I: Functionals defined on BD space, ibid. 31 (2004), 175-199.

[9] -, General method of regularization. III: The unilateral contact problem, ibid. 31 (2004), 473-492. 
[10] J. L. Bojarski, Regularization of noncoercive constraints in Hencky plasticity, ibid. 32 (2005), 327-340.

[11] G. Bouchitté and M. Valadier, Integral representation of convex functionals on a space of measures, J. Funct. Anal. 80 (1988), 398-420.

[12] N. Bourbaki, Espaces Vectoriels Topologiques, Éléments de mathématique, Livre V, Hermann, Paris, 1966.

[13] M. Carriero, A. Leaci and F. Tomarelli, Free gradient discontinuities, in: Calculus of Variations, Homogenization and Continuum Mechanics, G. Bouchitté et al. (eds.), Ser. Adv. Math. Appl. Sci. 18, World Sci., Singapore, 1994, 131-147.

[14] M. Costabel, M. Dauge and S. Nicaise, Singularities of Maxwell interface problems, Math. Model. Numer. Anal. 33 (1999), 627-649.

[15] N. Dunford and J. T. Schwartz, Linear Operators, Part I, Interscience, New York, 1958.

[16] C. Ebmeyer, J. Frehse and M. Kassmann, Boundary regularity for nonlinear elliptic systems: applications to the transmission problem, in: Geometric Analysis and Nonlinear Partial Differential Equations, S. Hildebrandt et al. (eds.), Springer, 2002, 505-517.

[17] I. Ekeland and R. Temam, Convex Analysis and Variational Problems, NorthHolland, Amsterdam, 1976.

[18] R. Engelking, General Topology, PWN-Polish Sci. Publ., Warszawa, 1977.

[19] A. Friedman and Y. Liu, Propagation of cracks in elastic media, Arch. Ration. Mech. Anal. 136 (1996), 235-290.

[20] E. Giusti, Minimal Surfaces and Functions of Bounded Variation, Monogr. Math. 80, Birkhäuser, 1984.

[21] R. Hardt and D. Kinderlehrer, Elastic plastic deformation, Appl. Math. Optim. 10 (1983), 203-246.

[22] D. Knees, Regularity results for transmission problems for the Laplace and Lamé operators on polygonal or polyhedral domains, Bericht SFB 404 2002/10, Universität Stuttgart, 2002.

[23] - On the regularity of weak solutions of quasi-linear elliptic transmission problems on polyhedral domains, Z. Anal. Anwend. 23 (2004), 509-546.

[24] R. Kohn and R. Temam, Dual spaces of stresses and strains with applications to Hencky plasticity, Appl. Math. Optim. 10 (1983), 1-35.

[25] J. A. König, Shakedown of Elastic-Plastic Structures, PWN-Polish Sci. Publ., Warszawa, and Elsevier, Amsterdam, 1987.

[26] W.-B. Liu, Degenerate quasilinear elliptic equations arising from bimaterial problems in elastic-plastic mechanics, Nonlinear Anal. 35 (1999), 517-529.

[27] D. Mercier, Minimal regularity of the solutions of some transmission problems, Math. Methods Appl. Sci. 26 (2003), 321-348.

[28] D. Mercier and S. Nicaise, Minimal regularity of the solutions of some boundary value problems of Signorini's type in polygonal domains, Math. Nachr. 278 (2005), 692-702.

[29] J. J. Moreau, Champs et distributions de tenseurs déformation sur un ouvert de connexité quelconque, Travaux Sém. Anal. Convexe 6 (1976), exp. 5, 23 pp.

[30] S. Nicaise and A.-M. Sänding, General interface problems I, Math. Methods Appl. Sci. 17 (1994), 395-429.

[31] —, - General interface problems II, ibid., 431-450.

[32] -, - , Transmission problems for the Laplace and elasticity operators: Regularity and boundary integral formulation, Math. Models Methods Appl. Sci. 9 (1999), $855-898$. 
[33] M. Petzoldt, Regularity results for Laplace interface problems in two dimensions, Z. Anal. Anwend. 20 (2001), 431-455.

[34] R. T. Rockafellar, Integral functionals, normal integrands and measurable selections, in: Nonlinear Operators and the Calculus of Variations, Lecture Notes in Math. 543, Springer, Berlin, 1975, 157-207.

[35] G. A. Seregin, Two dimensional variational problems in plasticity, Izv. Math. 60 (1996), 179-216.

[36] R. Temam, Mathematical Problems in Plasticity, Gauthier-Villars, Paris, 1985.

[37] R. Temam and G. Strang, Functions of bounded deformation, Arch. Ration. Mech. Anal. 75 (1980), 7-21.

[38] - , - Duality and relaxation in the variational problems of plasticity, J. Mécanique 19 (1980), 493-527.

[39] F. Tomarelli, Signorini problem in Hencky plasticity, Ann. Univ. Ferrara Sez. VII Sci. Mat. 36 (1990), 73-84.

[40] M. Valadier, Multi-applications mesurables à valeurs convexes compactes, J. Math. Pures Appl. 50 (1971), 265-297.

Jarosław L. Bojarski

Department of Applied Mathematics

Warsaw University of Life Sciences - SGGW

Nowoursynowska 159

02-787 Warszawa, Poland

E-mail: jaroslaw_bojarski@sggw.pl

Received on 13.1.2009;

revised version on 4.3.2011 
